\title{
Water and Volatiles in the Outer Solar System
}

\author{
O. Grasset ${ }^{1} \cdot$ J. Castillo-Rogez ${ }^{2} \cdot$ T. Guillot ${ }^{3}$. \\ L.N. Fletcher ${ }^{4}$ F. Tosi ${ }^{5}$
}

Received: 17 November 2016 / Accepted: 4 August 2017 / Published online: 29 August 2017

(C) Springer Science+Business Media B.V. 2017

\begin{abstract}
Space exploration and ground-based observations have provided outstanding evidence of the diversity and the complexity of the outer solar system. This work presents our current understanding of the nature and distribution of water and water-rich materials from the water snow line to the Kuiper Belt. This synthesis is timely, since a thorough exploration of at least one object in each region of the outer solar system has now been achieved. Next steps, starting with the Juno mission now in orbit around Jupiter, will be more focused on understanding the processes at work than on describing the general characteristics of each giant planet systems.
\end{abstract}

The Delivery of Water to Protoplanets, Planets and Satellites

Edited by Michel Blanc, Allessandro Morbidelli, Yann Alibert, Lindy Elkins-Tanton, Paul Estrada,

Keiko Hamano, Helmut Lammer, Sean Raymond and Maria Schönbächler

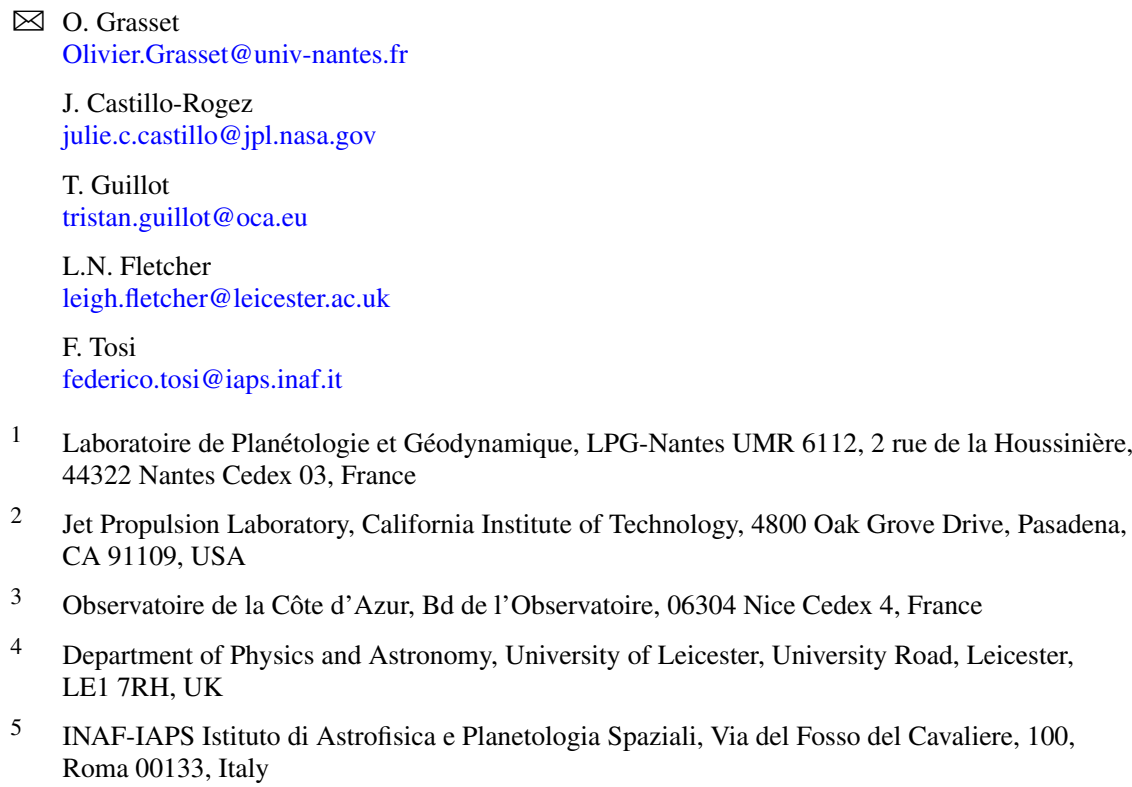


This review is organized in three parts. First, the nature and the distribution of water and volatiles in giant and intermediary planets are described from their inner core to their outer envelopes. A special focus is given to Jupiter and Saturn, which are much better understood than the two ice giants (Uranus and Neptune) thanks to the Galileo and Cassini missions. Second, the icy moons will be discussed. Space missions and ground-based observations have revealed the variety of icy surfaces in the outer system. While Europa, Enceladus, and maybe Titan present past or even active tectonic and volcanic activities, many other moons have been dead worlds for more than 3 billion years. Ice compositions found at these bodies are also complex and it is now commonly admitted that icy surfaces are never composed of pure ices. A detailed review of the distribution of non-ice materials on the surfaces and in the tenuous atmospheres of the moons is proposed, followed by a more focused discussion on the nature and the characteristics of the liquid layers trapped below the cold icy crusts that have been suggested in the icy Galilean moons, and in Enceladus, Dione, and Titan at Saturn. Finally, the recent observations collected by Dawn at Ceres and New Horizons at Pluto, as well as the state of knowledge of other transneptunian objects, are summarized, and complete this overview of the nature and distribution of ice-rich material in the outer solar system.

Keywords Water and volatiles · Giant and Intermediate planets · Giant moons and small bodies $\cdot$ Chemistry and Physics

\section{Introduction}

The outer solar system hosts a significant amount of water and volatiles, about half of the total mass of material once hydrogen and helium have been excluded, mostly located in the four outer planets. Beyond the "snow line" or "frost line" located at roughly 2.7 AU from the Sun, where radiation equilibrium temperature drops below $\sim 150 \mathrm{~K}$, water ice is stable over geologic timescales on the surface of airless bodies. The dwarf planet Ceres, the most massive object in the main asteroid belt, is located at $\sim 2.77$ AU from the Sun and has a low bulk density of $2.16 \mathrm{~g} \mathrm{~cm}^{-3}$ that reveals an icy mantle, while water ice was discovered only in a few specific places at the surface (Combe et al. 2016, 2017). At larger heliocentric distances, water ice is a major constituent of the surface and interior of the satellites revolving around the outer planets, and even more it dominates the bulk composition of the Kuiper Belt Objects (KBO). The abundance of water in the deep interior of both giant and intermediary planets is also ascertained, even if its amount is still poorly constrained. In the following sections, it is not only the water molecule but also the volatiles and the organic matter that will be studied for each planetary object in the outer solar system. Water ices can exist in several solid phases depending on temperature and pressure conditions (crystalline versus amorphous, low or high pressure polymorphs from the surface to the deep interiors of planetary objects), but also in the liquid state at least in a few icy moons of Jupiter and Saturn, and finally in the gaseous state especially in the exospheres of the small bodies and the deep atmospheres of the giant planets. In addition, the water ice is rarely found in a pure state. Water is indeed easily mixed with other species, either in a hydrated form or in a clathrate-hydrate structure. The pressure and temperature conditions, the nature of the volatiles as well as their abundance with respect to the water compound, influence the nature of the water phases that can be found. This diversity of components and phases will be explored from the asteroid belt to the Kuiper belt. For each domain, a discussion based on the most recent discoveries issued from recent space missions has been chosen.

Even if our current knowledge of giant and intermediary planets is subject to numerous uncertainties, especially in term of atmospheric/interior structure and compositions, previ- 
ous space missions and ground-based observations have provided sufficient information to understand their main characteristics. This will be discussed in Sect. 2 first by looking at the deep interior of these planets (Sect. 2.1), and second by exploring the composition and the dynamics of their outer envelopes (Sect. 2.2). Although volatile species are only found in trace quantities within the gaseous atmospheres of the four outer planets, their abundance and spatial distribution play a vital role in shaping the dynamics, clouds, and general circulation of the visible atmosphere, from the churning cloud decks of the troposphere through to the stable middle atmosphere. Spectroscopic measurements, combined with in situ sampling by the Galileo probe at Jupiter, have revealed that the most cosmogenically-abundant elements (carbon, oxygen, nitrogen, sulphur) are present within the gas giants Jupiter and Saturn in their reduced, hydrogenated forms $\left(\mathrm{CH}_{4}, \mathrm{H}_{2} \mathrm{O}, \mathrm{NH}_{3}, \mathrm{H}_{2} \mathrm{~S}\right)$. To date, the colder atmospheric temperatures of Uranus and Neptune have prevented the direct detection of all but $\mathrm{CH}_{4}$ gas on the ice giants, although this is found to be greatly enhanced (by 30-50 times) over the solar composition. By comparison to their gas giant cousins, we might expect the other chemical species to be similarly enriched in the ice giants (Lunine 1993), and this is borne out by microwave and radio observations.

In Sect. 3, the icy moons of the outer planetary systems will be explored. The flybys of the Pioneer and Voyager space missions have revealed the diversity of icy moon surfaces, suggesting that each moon may have had a unique history. This complexity and variety of features have been highlighted since then by the Galileo mission at Jupiter, the CassiniHuygens mission at Saturn, Dawn at Ceres, and finally New Horizons at Pluto and beyond. It is beyond the scope of this paper to describe the numerous patterns of all the icy moons in detail-the giant planets possess a very large number of moons ( $>67,>52,>27,>14$ for Jupiter, Saturn, Uranus, and Neptune, respectively), each of them being unique. Detailed descriptions can be found in the literature if not for all but for most of them, e.g., in Bagenal et al. (2006) for the jovian moons and in Dougherty et al. (2009) for the saturnian moons. In this paper, the focus will be first on the nature, the origin, and the distribution of the volatiles detected both in the tenuous atmospheres and on the surfaces of the moons. Second, we will be focused on the deep liquid reservoirs existing in a few moons only. The large amount of water available in the outer solar system implies that the hydrosphere can be extremely thick in differentiated bodies, namely several hundreds of kilometers in the case of the largest moons. In the Pioneer and Voyager era, it was commonly admitted that due to the very cold environment and to the vigorous convection within icy layers, icy satellites had to be entirely solid (Consolmagno and Lewis 1978; Reynolds and Cassen 1979). But it was also suggested that these bodies could host a deep ocean (Cassen et al. 1982). It turned out, thanks to the observations returned by the Galileo and Cassini missions, that some moons could indeed preserve deep liquid reservoirs until present. Over the past two decades many studies have explored the properties and evolution of icy satellite shells. These are driven on one side by the dynamical processes (especially tidal forcing-Hussmann et al. 2010) and on the other side by the amount and nature of the volatiles compounds trapped in the moons (Fortes and Choukroun 2010). One may have the feeling that deep liquid reservoirs are indeed very common and expected in all midsized and large icy moons. This idea was explored in the past (see for example Hussmann et al. 2006), especially knowing that it is indeed the case for at least one of the midsized moons, Enceladus (Cadek et al. 2016) and suspected in Dione (Beuthe et al. 2016). Nonetheless, there is no way to ascertain such a hypothesis at the present time in absence of geophysical observations at most of these bodies. The only moons, for which a liquid layer is envisaged (in terms of expected composition, depth and thickness, and habitability potential), are the following: Europa, Ganymede, and Callisto at Jupiter; Titan and Enceladus at Saturn. To our knowledge, those are the moons for which 
significant evidence of the existence of liquid layers has been ascertained by geophysical measurements.

The last section will be devoted to the special cases of dwarf planets found in or believed to have originated from the outer solar system. This paper will be focused on the cases where significant and recent progresses have been made thanks to the Dawn and New Horizon missions.

\section{Giant and Intermediate planets}

First, the deeper parts of the planets will be described. Second, the outer envelopes, on which many more constraints and measurements are available, will be characterized. Our goal is not to provide an exhaustive and complete description of all the layers of each planet, but rather to provide a state-of-the-art discussion focused on the distribution, the origins, and the transfer processes of water and volatiles trapped in these planets. Two space missions, Cassini orbiting Saturn since 2004, and Juno orbiting Jupiter since mid-2016, provide further important data to constrain the deep interiors of the giant planets. Both use polar eccentric orbits with periapses located extremely close to the planets' atmosphere in order to make high accuracy determination of their gravity fields. In addition, Juno will probe the deep atmosphere down to about 100 bars using microwave antennas, measuring ammonia abundances and providing constraints on the deep abundance of water in the planet. This will be extremely important to link measurements made in the atmosphere (see Sect. 2.2) to those inferred for the deep interior (see Sect. 2.1) and to really constrain the amount of heavy elements and ices present in the planets, both in their central cores and in their envelopes.

\subsection{Ices and Volatiles in Giant Planet Interiors}

In this section, the composition and the deep internal structure of the four outer planets are described, with a special focus on the amount and distribution of "ices" 1 within the cores and the inner envelopes. A tentative description of the origin and evolution of these deep layers, especially regarding the distribution of the ices, is also provided.

\subsubsection{Jupiter and Saturn}

Jupiter and Saturn are composed primarily of hydrogen and helium but they most likely contain more volatiles than all the other objects in the solar system added together, the Sun excluded. Jupiter is thought to contain between 10 and 40 Earth masses in heavy elements (i.e., all elements except hydrogen and helium) and Saturn between 20 and 30 Earth masses (Guillot 2005; Nettelmann et al. 2012; Helled and Guillot 2013; Hubbard and Militzer 2016; Miguel et al. 2016). Even larger quantities of heavy elements may be present in the interior if the structure departs significantly from an adiabat (Leconte and Chabrier 2012). We do not know what fraction of these heavy elements are in the form of "ices": The problem is highly degenerate and because all heavy elements are significantly denser than hydrogen and helium, their contribution in terms of global density is very similar. It is natural and convenient to assume that the respective abundance ratio between heavy elements remain solar. The standard mix of "ices" (including $\mathrm{H}_{2} \mathrm{O}, \mathrm{CH}_{4}$ and $\mathrm{NH}_{3}$ ices) and "rocky material"

\footnotetext{
${ }^{1}$ In this section, the term "ices" stands for $\mathrm{H}_{2} \mathrm{O}, \mathrm{CO}, \mathrm{NH}_{3}$ (and possibly $\mathrm{CO}$ and $\mathrm{CO}_{2}$ ) because of their condensation in the form of solid ices at low temperature in the protosolar disk. However, these compounds become fluid in the high-pressure and high-temperature interiors of giant planets.
} 
(including silicates and iron, called silicate-rich hereafter) obtained from the condensation of a solar composition gas at the distance of Jupiter is $2 / 3$ ices and $1 / 3$ silicate-rich (Lodders 2003). This implies that between 20 and 50 Earth masses of ices could be hidden inside Jupiter and Saturn.

These heavy elements (and ices) are believed to be partly hidden as a central core and partly distributed in the envelopes of the planets. Constraints from gravitational moments indicate that the mass of the central core is less than about $20 \mathrm{M} \bullet$ in both Jupiter and Saturn with values which depend significantly on the equations of state of hydrogen used (Nettelmann et al. 2012; Militzer and Hubbard 2013; Hubbard and Militzer 2016; Miguel et al. 2016) and on the location at which helium separates out from hydrogen (Helled and Guillot 2013).

This central core is an important ingredient for the formation of a giant planet: The accretion scenario shows that a giant planet can form by the collapse of a hydrogen-helium envelope around a high-density core estimated to be around $10 \mathrm{M}_{\text {ठ }}$ (e.g., Mizuno 1980; Pollack et al. 1996; Wuchterl et al. 2000; Alibert et al. 2005). Another class of solution invokes a direct instability of the gas disk followed by the capture of heavy elements (e.g., Boley et al. 2010; Nayakshin et al. 2014). In all cases, the presence of an abundant source of solids in the outer solar system in the form of dust grains and pebbles appears to be essential for the formation of giant planets as it helps building a core quickly before the removal of the gas disk (e.g., Lambrechts and Johansen 2012; Guillot and Gautier 2015; Levison et al. 2015).

It is presently unclear how to account for the large masses in heavy elements in the envelopes of Jupiter and Saturn and for the enrichment of their atmospheres (see section hereafter). This must be done early, the giant planets becoming rapidly very efficient scatterers of planetesimals (Matter et al. 2009). One possibility is to invoke an efficient capture of planetesimals during the growth of the envelope phase (e.g., Alibert et al. 2005). Another option is that the envelopes may have been enriched by the progressive erosion of the core (Guillot et al. 2004) or generally by upward mixing of material present in the lower envelope (Vazan et al. 2016). Finally the protosolar disk itself may have been enriched in heavy elements by selective photo-evaporation at the time of the formation of the giant planets (Guillot and Hueso 2006; Monga and Desch 2015).

\subsubsection{Uranus and Neptune}

With masses of $14 \mathrm{M}_{+}$and $17 \mathrm{M}_{+}$for densities of $1.2 \mathrm{~g} / \mathrm{cm}^{3}$ and $1.7 \mathrm{~g} / \mathrm{cm}^{3}$, respectively, Uranus and Neptune look like failed giant planets. While they seem relatively similar, Neptune's larger mean density compared to Uranus indicates a slightly different composition: either more heavy elements compared to hydrogen and helium or a larger rocky to icy materials ratio. The gravitational moments impose that the density profiles lie close to that of 'ices' (a mixture initially composed of, for example, $\mathrm{H} 2 \mathrm{O}, \mathrm{CH} 4$, and NH3 but that rapidly becomes an ionic fluid of uncertain chemical composition in the planetary interior), except in the outermost layers, which have a density closer to that of hydrogen and helium (Marley et al. 1995; Podolak et al. 2000). Most models of these planets assume the presence of three layers, a central core (magnesium-rich silicate and iron material), an ice layer, and a hydrogen-helium gas envelope (e.g., Helled et al. 2011; Nettelmann et al. 2013).

According to the models of Nettelmann et al. (2013), Uranus contains a minimum of 1.8-2.2 $\mathrm{M}_{+}$of hydrogen and helium and Neptune 2.7-3.3 $\mathrm{M}_{+}$. The global ice to silicaterich ratio that is derived is very high (19-36) in Uranus, while Neptune has a wide range of solutions from 3.6 to 14 . These values are much larger than the canonical ice to silicate-rich 
ratio of 2-3 for the protosun that accounts for the abundances of all elements condensing at low temperatures ('ices') versus that of more refractory elements ('silicate-rich'). The fact that either planet would have accreted much less silicate-rich phases than ices is puzzling and unexplained by formation models. It is probably an artefact from assuming that ices are confined to the envelope and silicates and iron to the core.

In fact, it is likely that all present models of Uranus and Neptune are inadequate because of the assumption of an adiabatic temperature structure across interfaces with different compositions. The high abundance of methane is shown to inhibit convection in its cloud formation region (between 1 and 2 bar) and leads to a superadiabatic temperature gradient that was observed in Voyager radio-occultation data (Guillot 1995). If water is more than ten times solar, a similar but quantitatively stronger effect may occur, characterized by the presence of a deep radiative zone with a strong increase of the temperature with depth (Guillot 1995; Leconte et al. 2017). Still deeper, diffusive convection should occur in regions with compositional changes, also leading to a superadiabatic temperature gradient (e.g., Rosenblum et al. 2011). As in the case of Jupiter and Saturn (see Leconte and Chabrier 2012), this would lead to higher temperatures in the interior and very different constraints on the interior composition. The amount of rocky material required to fit the mean density and gravitational moments would certainly rise, potentially solving the ice to rocky material ratio problem.

\subsection{Water and Volatiles in the Gaseous Envelopes}

In this section, we review the present state of knowledge of the abundances of the simple molecules $\left(\mathrm{CH}_{4}, \mathrm{H}_{2} \mathrm{O}, \mathrm{NH}_{3}, \mathrm{H}_{2} \mathrm{~S}\right)$ and their isotopologues in the gaseous envelopes of the outer planets. We will describe their importance for cloud formation, their role in atmospheric dynamics, and isotopic ratios within these species as a constraint on planetary origins. We will also briefly review the present-day supply of oxygenated species to their upper atmospheres, via cometary impacts and influx from meteorites, satellites and rings.

\subsubsection{Volatiles and Clouds}

The vertical distribution of volatile species (methane, ammonia, hydrogen sulphide and water) is governed by a variety of processes, as depicted in Fig. 1. Thermochemical equilibrium modelling (e.g., Weidenschilling and Lewis 1973) provides a first estimate of the importance of condensation, whereby a cloud deck condenses to separate a well-mixed internal reservoir of the species at depth from a higher-altitude region where the volatile follows its own relative humidity curve. The altitudes of the cloud decks are governed by the abundance of the species in question, their volatility (i.e., condensation point) and the temperature structure of the environment.

Methane Methane is the most volatile species, condensing only on the ice giants, Uranus and Neptune, between the $\sim 1$ bar pressure level and the tropopause, meaning that the topmost clouds are expected to consist of methane ices. On the gas giants, where temperatures never get this cold, methane is expected to be well-mixed everywhere, and only begins to decrease in abundance at high stratospheric altitudes due to the effects of photochemistry and diffusion (Moses et al. 2005). Indeed, the presence of methane in the upper tropospheres and stratospheres of all four giants has two notable effects. Firstly, the stratospheres are replete with hydrocarbon species derived from the photolysis of $\mathrm{CH}_{4}$ (see Moses et al. 2005 for a review). Secondly, the abundance of methane has been used as an estimate of the bulk carbon enrichment of all four giants, increasing from $\sim 5 \times$ enrichment for Jupiter 
(a) Jupiter

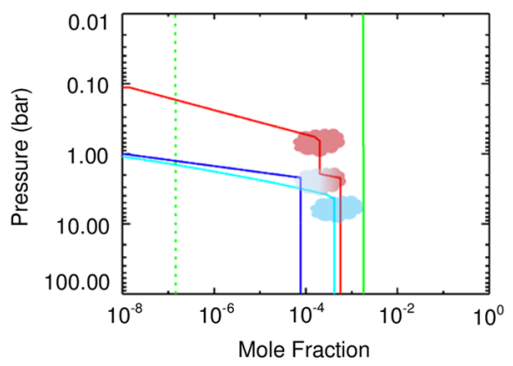

(c) Uranus

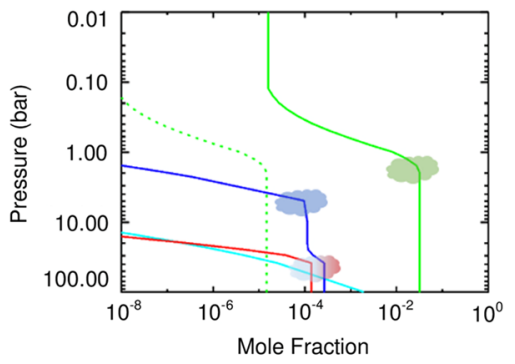

(b) Saturn

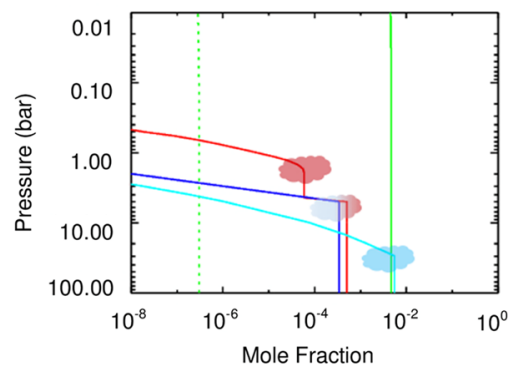

(d) Neptune

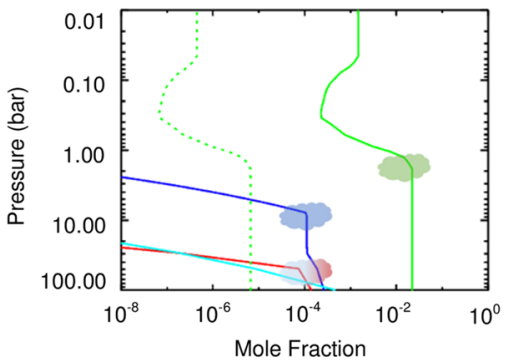

Fig. 1 Observed and modeled vertical distributions of volatiles on all four giant planets, including $\mathrm{CH}_{4}$ (green), $\mathrm{NH}_{3}$ (red), $\mathrm{H}_{2} \mathrm{~S}$ (blue), $\mathrm{H}_{2} \mathrm{O}$ (cyan) and $\mathrm{CH}_{3} \mathrm{D}$ (dotted green). Approximate locations of condensate clouds are also shown $\left(\mathrm{CH}_{4}\right.$ ice in green, $\mathrm{NH}_{3}$ ice in red, $\mathrm{H}_{2} \mathrm{~S}$ in blue, $\mathrm{NH}_{4} \mathrm{SH}$ as a hybrid of red/blue, and $\mathrm{H}_{2} \mathrm{O}$ ice in cyan). $\mathrm{CH}_{4}$ distributions are from Galileo (Jupiter), Cassini (Saturn), Spitzer (Uranus) and AKARI (Neptune) analyses. $\mathrm{NH}_{3}, \mathrm{H}_{2} \mathrm{~S}$ and $\mathrm{H}_{2} \mathrm{O}$ are from combinations of direct observation (Galileo), remote sensing of the $p<2$ bar range (Jupiter and Saturn), and cloud condensation modelling following Irwin et al. (2009) - this allows us to see the interplay between $\mathrm{NH}_{3}$ and $\mathrm{H}_{2} \mathrm{~S}$ in the top two cloud layers, where cloud condensation causes discrete steps in the profiles. The true vertical distributions of these species for $p>2$ bar remain to be properly quantified by measurements (see main text)

(Wong et al. 2004a, 2004b), to $\sim 10 \times$ enrichment for Saturn (Fletcher et al. 2009), to $\sim 90 \times$ enrichment for Uranus (Sromovsky et al. 2011) and Neptune (Karkoschka and Tomasko 2011). Given that Jupiter showed uniform enrichments of all elements by $2-5$ over protosolar composition (excluding oxygen and neon), we might expect similar enrichments on all the giants, with a greater proportion of heavy elements in the ice giants than the gas giants due to their increased core-to-envelope ratios (Owen and Encrenaz 2003). We note, however, that methane gas appears to vary with latitude on the ice giants (Sromovsky et al. 2011; Karkoschka and Tomasko 2011), being more abundant at low latitudes than the poles, highlighting the importance of atmospheric dynamics in governing the abundances of these species.

Ammonia and Hydrogen Sulphide Below the methane clouds, the condensation levels of ammonia $\left(\mathrm{NH}_{3}\right)$ and hydrogen sulphide $\left(\mathrm{H}_{2} \mathrm{~S}\right)$ is made more complex by a two-component reaction that produces a solid-phase cloud of $\mathrm{NH}_{4} \mathrm{SH}$ (ammonium hydrosulphide), which either serves to consume all of the $\mathrm{NH}_{3}$ or all of the $\mathrm{H}_{2} \mathrm{~S}$, depending on which species is more abundant (i.e., depending on the N/S ratio). If the giant planets showed a steady enrichment of the solar composition, then $\mathrm{NH}_{3}$ should always be more abundant than $\mathrm{H}_{2} \mathrm{~S}(\mathrm{~N} / \mathrm{S}>1)$, so that there would be $\mathrm{NH}_{3}$ left over to form the topmost condensation cloud. However, this has 
not been observationally verified on all four giants. On Jupiter, the in situ measurements of Galileo demonstrated that the $\mathrm{NH}_{3}$ and $\mathrm{H}_{2} \mathrm{~S}$ abundances were both enriched over the solar composition in the 8-12 bar range, at least within the descent site in the Northern Equatorial Belt. $\mathrm{NH}_{3}$ was found to be more enriched than $\mathrm{H}_{2} \mathrm{~S}(\mathrm{~N} / \mathrm{S}>$ solar), implying that all of the $\mathrm{H}_{2} \mathrm{~S}$ would be consumed by the combination reaction. Recent microwave mapping (de Pater et al. 2016) has confirmed that $\mathrm{NH}_{3}$ enrichments are present in some locations on Jupiter, but that this is not the case over the whole planet. Jupiter's topmost cloud, near 800 mbar and $\sim 150 \mathrm{~K}$, should be composed of $\mathrm{NH}_{3}$ ice, with a deeper cloud of $\mathrm{NH}_{4} \mathrm{SH}$ solid near $\sim 2$ bar and $210 \mathrm{~K}$ (West et al. 2004). Indeed, $\mathrm{NH}_{3}$ ice has been spectroscopically identified in regions of powerful cloud activity on Jupiter (Baines et al. 2002; Wong et al. 2004b)

Unfortunately, the bulk abundances of $\mathrm{NH}_{3}$ and $\mathrm{H}_{2} \mathrm{~S}$ remain poorly understood on the other giants for two reasons - the lack of in situ measurement, and the colder temperatures pushing these species to deeper and more inaccessible pressures. Infrared (IR) observations, such as those from Cassini, ISO, Voyager and Spitzer, are sensitive to ammonia absorption in the upper tropospheres, above the main cloud decks in the region where $\mathrm{NH}_{3}$ is declining with height. $\mathrm{NH}_{3}$ dominates the 5-, 10- and 100- $\mu \mathrm{m}$ regions in Jupiter's spectrum (Fouchet et al. 2000; Achterberg et al. 2006) and has a clear influence on Saturn (de Graauw et al. 1997), but $\mathrm{NH}_{3}$ estimates from these observations are only lower limits on the bulk $\mathrm{NH}_{3}$ content. $\mathrm{No} \mathrm{NH}_{3}$ signatures have ever been identified on Uranus and Neptune in this range, and no infrared $\mathrm{H}_{2} \mathrm{~S}$ gas signatures have ever been observed. Microwave observations hold more promise, sensing the deeper troposphere by utilizing a broad $\mathrm{NH}_{3}$ absorption band near 1-cm wavelength to sense below the clouds. Juno's Microwave Radiometer (MWR), which successfully began operations at Jupiter in 2016, will be the first to map this absorption from a planetary spacecraft, but all other inferences come from challenging ground-based microwave observations. $\mathrm{H}_{2} \mathrm{~S}$, in particular, has only a broad-band influence over the microwave spectrum, so the abundance remains highly uncertain. On Saturn, the only reported measurement (Briggs and Sackett 1989) suggests a large enrichment of $\mathrm{H}_{2} \mathrm{~S}$ compared to $\mathrm{NH}_{3}$ (de Pater and Massie 1985; Janssen et al. 2013), suggesting N/S < solar. This controversial result is rarely incorporated into cloud-equilibrium modelling - either Saturn's abundance of $\mathrm{NH}_{3}$ is expected to be higher, or the abundance of $\mathrm{H}_{2} \mathrm{~S}$ is expected to be lower. Most authors assume a Jupiter-like vertical structure, with $\mathrm{NH}_{3}$ ice overlaying a cloud of solid $\mathrm{NH}_{4} \mathrm{SH}$. Recent spectroscopic observations during Saturn's springtime storm (Sromovsky et al. 2013) were consistent with the presence of $\mathrm{NH}_{3}$ ice.

The situation is even more challenging for Uranus and Neptune, where no $\mathrm{NH}_{3}$ or $\mathrm{H}_{2} \mathrm{~S}$ signatures have been detected in the infrared. Microwave observations have suggested that Uranus' ammonia is strongly depleted in the 10-20 bar range with respect to solar (de Pater and Massie 1985), whereas $\mathrm{H}_{2} \mathrm{~S}$ is much more enriched (by factors of 10-35, de Pater et al. 1991). Microwave observations of Neptune tell the same story (de Pater and Massie 1985; de Pater et al. 1991). The ammonia measurements on both planets could be upper limits, if all the $\mathrm{NH}_{3}$ were consumed by the formation of the $\mathrm{NH}_{4} \mathrm{SH}$ cloud near 40-50 bar, or if substantial amounts of $\mathrm{NH}_{3}$ were incorporated into an aqueous $\mathrm{NH}_{3}-\mathrm{H}_{2} \mathrm{O}$ cloud at greater depths. Alternatively, the ice giants could genuinely be nitrogen-poor when compared to the gas giants, due to the different source regions for the icy materials from which they accreted. Or maybe ice giant nitrogen exists mainly as $\mathrm{N}_{2}$, a disequilibrium species in the observable atmospheres that is difficult to detect spectroscopically. In any case, the N/S $<1$ implied by these measurements suggests that the ice giants differ from the gas giants in that an $\mathrm{H}_{2} \mathrm{~S}$ cloud forms near 4-8 bar above the $\mathrm{NH}_{4} \mathrm{SH}$ solid at 40-50 bar, rather than having $\mathrm{NH}_{3}$ ice as the topmost cloud (as shown in Fig. 1). 
Water Below the carbon, nitrogen and sulphur-based clouds, the water cloud is the most challenging to observe, and yet the most important for constraining theories of atmospheric dynamics and planetary origins. The condensation of the water ice cloud, near 5 bar on Jupiter, 10 bar on Saturn, and hundreds of bars on the ice giants, loosely defines the base of the weather layer and separates it from the deep interior. Identifying this cloud in remote sensing data is challenging, given the wide range of gaseous and aerosol absorptions overlying this cloud deck, and only on Jupiter has a putative cloud been identified at the right altitude to be composed of $\mathrm{H}_{2} \mathrm{O}$ ice (Nixon et al. 2001; Banfield et al. 1998; Bjoraker et al. 2015). Lighting activity, related to charge-separation in the water ice cloud, has also been observed directly on Jupiter (Little et al. 1999; Gierasch et al. 2000). Spectroscopically-identifiable water ice has only ever observed in Voyager data near $44 \mu \mathrm{m}$ (Simon-Miller et al. 2000), and is not widespread. Gaseous water, residing above the cloud deck and following a saturated vapor pressure curve, influences the 5$\mu \mathrm{m}$ spectrum of Jupiter (measured from Galileo and Cassini data by Roos-Serote et al. 2000; Giles et al. 2015). However, precise estimates of its abundance and vertical distribution are extremely challenging. Given that the Galileo probe measurements showed that the water abundance was still increasing, even when they ceased near 22 bar (Niemann et al. 1998; Wong et al. 2004a, 2004b), the question of Jupiter's deep water abundance remains an unresolved problem. Juno's Microwave Radiometer aims to determine both the deep $\mathrm{H}_{2} \mathrm{O}$ and $\mathrm{NH}_{3}$ abundances to 100 bar of pressure.

For Saturn, the powerful 2010 storm system featured spectroscopic signatures that could be reproduced by a mixture of water ice, ammonia ice and $\mathrm{NH}_{4} \mathrm{SH}$ (Sromovsky et al. 2013), although this depends on the assumed optical properties of the particles. Upper tropospheric signatures of gaseous water have been observed by ISO at $5 \mu \mathrm{m}$ (de Graauw et al. 1997), but Cassini lacked the spectral resolution to map these across the planet, and ground-based observations must deal with considerable contamination from terrestrial water. Given the challenge of direct measurements of Saturn's water (Mousis et al. 2014), the abundances of disequilibrium species $\left(\mathrm{CO}, \mathrm{PH}_{3}\right.$, etc.) that react chemically with water can be used to infer the abundance indirectly (Visscher et al. 2010). Although these typically predict a supersolar water enrichment, there remains huge uncertainties in the water abundance due to the need to assume a vertical mixing rate in the calculations and the criticality of the choice of reaction network.

Despite being known as ice giants, the water on Uranus and Neptune is even more inaccessible, with liquid and ice clouds forming at hundreds of bars of pressure, and no detections of gaseous or solid water to date (with the exception of exogenic sources, see below). Indirect estimates based on the CO abundance (Lodders and Fegley 1994; Marten et al. 1993; Cavalié et al. 2014) suggest that the O/H enrichment of the ice giants may reach factors of several hundred. Microwave observations of Uranus suggested a deep absorbing layer that could have been related to a water cloud (Hofstadter and Muhleman 1989; Hofstadter and Butler 2003), whereas analysis of Neptune microwave data by de Pater et al. (1991) simply assumed a water abundance. Indeed, even the microwave spectra have a limited sensitivity to this deep water, but assessing its abundance should be a primary goal of any future ice giant mission.

\subsubsection{Spatial Distribution and Atmospheric Dynamics}

Although a detailed review of giant planet dynamics is beyond the scope of this book (see Ingersoll et al. 2004; Del Genio et al. 2009 for comprehensive reviews), we note that the distribution and condensation of volatiles plays a crucial role in shaping the general circulation and 'weather' of these giant planet atmospheres. Each condensate cloud forms a 
molecular weight gradient in the planet's density structure, serving to inhibit convective motions through that layer (e.g., Stoker 1986; Sugiyama et al. 2014). They can thus serve to isolate and stabilize different vertical regions, permitting circulation cells with differing directions between the cloud layers. However, strong vertical motions can overcome these barriers, resulting in powerful convective activity being witnessed in the topmost cloud decks, from billowing storm activity and their associated lightning on the gas giants (e.g., Ingersoll and Vasavada 2000; Gierasch et al. 2000; Sanchez-Lavega et al. 2011; Fischer et al. 2011; Fletcher et al. 2011a, 2011b) to large storm eruptions on the ice giants (e.g., de Pater et al. 2015). These storms are superimposed onto an intermediatescale banded structure (with convective storm activity preferentially occurring in cyclonic, cloud-free belts) and, possibly, a large scale circulation pattern with air moving from the seasonally-dependant sub-solar point to higher latitudes (e.g., Conrath et al. 1990). Vertical and horizontal motions associated with these circulation patterns will affect atmospheric temperatures, cloud formation, and the abundances of these species, potentially explaining why ices of ammonia and water have only been identified in regions of powerful storm activity, and why methane exhibits strong equator-to-pole contrasts on the ice giants.

This energy cascade from the smallest to the largest scales (or vice versa) is a topic of active research, but the condensation of volatiles-primarily water-provides the energy source through latent heating to drive the convective motions. Once a parcel of air is forced upwards, the water condensation will provide energy that makes the parcel buoyant, able to penetrate through the overlying stable layers until it reaches the stably-stratified upper troposphere. Here the divergent air cools, condensates begin to precipitate out as rain or snow, to re-evaporate again in the deep atmosphere. Energy can penetrate higher into the stratosphere in the form of waves spawned by this powerful convection (Fletcher et al. 2012), serving to modify the circulation of the middle-atmosphere. Giant planet volatiles are therefore directly connected to the dynamic motions shaping the ever-changing appearance of their atmospheres.

\subsubsection{Bulk Abundances and Isotopic Composition}

Recent reviews of the bulk elemental and isotopic compositions of the giant planets can be found in Mousis et al. (2014) and Guillot and Gautier (2015), here we summarize the key findings for the four key elements $(\mathrm{C}, \mathrm{N}, \mathrm{S}$, and $\mathrm{O})$. However, we note that the atmospheric and interior compositions may be decoupled by phase transitions, cloud formation, chemical reactions and phase separation, implying that the atmospheric composition may not be a good representation of the bulk (Guillot and Gautier 2015). Furthermore, the fact that most remote sensing measures volatile abundances above their primary condensation altitudes means that large uncertainties are involved. As described above, $\mathrm{C} / \mathrm{H}$ appears to be enriched over protosolar composition by $5 \times$ on Jupiter, $10 \times$ on Saturn, and $90 \times$ on Uranus and Neptune. $\mathrm{N} / \mathrm{H}$ appears to be more enriched on Jupiter $(4 \times$, as measured by the Galileo probe, Wong et al. 2004a, 2004b) than on Saturn (3×, determined from Cassini remote sensing, Fletcher et al. 2009, 2011a, 2011b), although this could be a result of an inaccessible reservoir of nitrogen below the clouds. Conversely, microwave modelling by de Pater and Massie (1985) suggested a substantial sub-solar N/H on the ice giants, which may also be associated with sequestration in deeper cloud decks. Like carbon, $\mathrm{S} / \mathrm{H}$ is higher on Saturn $(8 \times$, based on the radio-wavelength measurements of Briggs and Sackett 1989) than on Jupiter (3×, based on the Galileo probe, Wong et al. 2004a, 2004b), and substantially enriched $(10-35 \times)$ on both ice giants (de Pater et al. 1991). Finally, the deep abundance of oxygen is a key unknown on all four giant planets, with indirect estimates of supersolar enrichments 
varying by an order of magnitude, from tens to hundreds of times solar. Given that trapping of volatile species within ices may have formed the building blocks of the protoplanetary cores, this uncertainty in the $\mathrm{O} / \mathrm{H}$ is a crucial problem to solve.

Measurements of isotopic ratios within these molecules, however, can provide further insights even if the absolute abundances remain unknown. This is because the isotopic fractionation is not expected to have changed over time and should be the same irrespective of the phase transitions and cloud condensation processes that may be occurring. The isotopic composition in the volatiles that are accessible to remote sensing include those in the primary atom $\left({ }^{13} \mathrm{C} /{ }^{12} \mathrm{C},{ }^{15} \mathrm{~N} /{ }^{14} \mathrm{~N}\right)$ and in hydrogen $(\mathrm{D} / \mathrm{H})$. There have been no measurements of the isotopic composition in sulphur or deep oxygen (see below for exogenic sources). Measurements of the ${ }^{13} \mathrm{C} /{ }^{12} \mathrm{C}$ ratio are available for Jupiter (Galileo probe, Atreya et al. 2003), Saturn (Cassini infrared measurements, Fletcher et al. 2009) and for Neptune (ground-based observations, Orton et al. 1992), and all show consistency with the terrestrial value of $89.9_{-2.4}^{+2.6}$, and therefore imply that the giants accreted from the same material as the Sun and that no further isotopic fractionation has taken place.

Deuterium has been more widely measured on the giant planets, in both pure $\mathrm{H}_{2}$ and in $\mathrm{CH}_{3} \mathrm{D}$, using infrared and sub-millimeter spectroscopy. The most recent gas giant values come from ISO (e.g., Lellouch et al. 2001) and Cassini (Fletcher et al. 2009), whereas the most recent ice giant values come from Herschel (Feuchtgruber et al. 2013), Spitzer (Orton et al. 2014a, 2014b), AKARI (Fletcher et al. 2010) and ground-based observations (Irwin et al. 2014). The results fall into two natural classes, with Jupiter and Saturn exhibiting approximately protosun values of $\mathrm{D} / \mathrm{H}\left(1.94 \times 10^{-5}\right)$, and Uranus and Neptune showing an enrichment of 2-3 compared to protosun values. In the case of Uranus and Neptune, this is considerably lower than what might be expected for an ice-dominated planet (assuming $\mathrm{D} / \mathrm{H}$ ratios in known ice sources in the solar system, such as cometary ices), prompting Feuchtgruber et al. (2013) to postulate that these worlds are in fact dominated by rocky material, rather than ices. However, alternative sources of protoplanetary ices with lower $\mathrm{D} / \mathrm{H}$ ratios than in comets, still awaiting identification, might explain this result. It is also possible, and even likely, that the ices in the interiors of Uranus and Neptune were only incompletely mixed, therefore preventing any such inference on the ice-to-rock ratio in the planetary interior. Furthermore, there are tantalizing hints that Saturn's D/H ratio is actually smaller than that of Jupiter (Fletcher et al. 2009), suggesting that the volatile reservoirs for Jupiter and Saturn were different, although this remains a topic of active study.

Further constraints on the volatile origins arise from the ${ }^{15} \mathrm{~N} /{ }^{14} \mathrm{~N}$ ratio measured in ammonia on Jupiter and Saturn. Molecular $\mathrm{N}_{2}$ is the dominant form of nitrogen in molecular clouds, but requires low environmental temperatures to trap within icy cages to form protoplanets. $\mathrm{NH}_{3}$, conversely, is easier to trap at warmer temperatures. Making use of the fact that ${ }^{15} \mathrm{~N}$ is enriched in nitrogen molecules compared to $\mathrm{N}_{2}$, the bulk ${ }^{15} \mathrm{~N} /{ }^{14} \mathrm{~N}$ ratio therefore reveals whether $\mathrm{N}_{2}$ or $\mathrm{NH}_{3}$ was the dominant carrier of nitrogen to the giant planets. Jupiter's ${ }^{15} \mathrm{~N} /{ }^{14} \mathrm{~N}$ has been measured in situ and remotely (Niemann et al. 1998; Owen et al. 2001; Fouchet et al. 2000, 2004; Fletcher et al. 2014) and was found to be considerably smaller than the terrestrial value, and is thought to be the same as that found in the protosun. Upper limits on Saturn's ${ }^{15} \mathrm{~N} /{ }^{14} \mathrm{~N}$ have been reported via ground-based spectroscopy (Fletcher et al. 2014), and found to be consistent with that of Jupiter. Both of these values are considerably smaller than the ${ }^{15} \mathrm{~N} /{ }^{14} \mathrm{~N}$ found in cometary molecules in the outer solar system. This suggests that molecular $\mathrm{N}_{2}$ dominates the nitrogen inventory of both gas giants, rather than ice-trapped $\mathrm{NH}_{3}$. This delivery could have been in the gas phase (i.e., along with the $\mathrm{H}_{2}$ and $\mathrm{He}$, although some method for enriching nitrogen must then be found) or trapped in icy planetesimals formed at cold, distant locations (and then 
transported inwards without vaporisation into the feeding zones of the giants). Furthermore, the Saturnian value is smaller than that of Titan (Niemann et al. 2010), suggesting different accretion reservoirs for the planet and its satellites.

\subsubsection{Exogenic Sources}

So far, we have discussed the vertical and horizontal distribution of volatiles that were incorporated into the planets during their formation. However, high-resolution spectroscopy over the past two decades have revealed a continuing source of volatile delivery-particularly oxygenated species - to their upper atmospheres (Feuchtgruber et al. 1997; Moses et al. 2000). This oxygen arises from a range of processes, including cometary impacts, diffusion of ring particles, debris from satellites, and the ablation of micrometeroids in the upper atmosphere. The subsequent chemistry gives rise to water, $\mathrm{CO}_{2}$ and $\mathrm{CO}$ detected as fine emission lines in the infrared and sub-millimeter spectrum, and measurements of their abundances and isotopic composition (including ${ }^{18} \mathrm{O} /{ }^{16} \mathrm{O}$ ) tell us about the present-day materials falling into the giants, rather than the origins of volatiles during planetary formation.

These stratospheric oxygen compounds were first studied by ISO (Feuchtgruber et al. 1997). The Shoemaker-Levy 9 cometary impact on Jupiter (1994) was a source of water and $\mathrm{CO}_{2}$ (Lellouch et al. 2002); the geysers of Enceladus may well provide water to Saturn's upper atmosphere (Hartogh et al. 2011). Spitzer has measured Uranus' $\mathrm{CO}_{2}$ (Orton et al. 2014b), and Uranus' CO was detected in fluorescence, its vertical distribution suggesting an external source (Encrenaz et al. 2004) which was more comprehensively studied by Herschel (Cavalié et al. 2014). Neptune's CO has also been revealed to have an external source (Hesman et al. 2007; Lellouch et al. 2010; Fletcher et al. 2010), possibly related to an ancient cometary strike, or to the slow rain of dust and micrometeorites.

These oxygen compounds can affect the stratospheric and ionospheric chemistry (particularly via the photolysis of $\mathrm{H}_{2} \mathrm{O}$, which then leads to $\mathrm{CO}$ and $\mathrm{CO}_{2}$ formation), and could lead to the formation of thin water ice clouds in the upper atmosphere (Moses et al. 2000). Future missions to Jupiter (e.g., JUICE) will make use of the distribution of this infalling watery debris to understand the properties of the stratosphere and the nature of the materials being accreted today.

\subsubsection{Summary}

Carbon, nitrogen, sulphur and oxygen are found in their reduced, hydrogenated forms in giant planet atmospheres, and condense (or react) to form discrete cloud decks in planetary atmospheres. The latent heat of water condensation plays a vital role in powering the dynamic activity which shapes the meteorology and climate of these worlds. Ammonia and methane ices dominate the upper clouds of the gas and ice giants, respectively, whereas the water clouds are deep and hidden from the view of remote sensing. This presents a significant challenge for assessing the chemical inventories of these worlds, as neither in situ probes nor remote sensing have penetrated the well-mixed, deep reservoirs for these species. A comparison of the bulk elemental composition and isotopic ratios on the four giant planets can be instructive in understanding the different volatile reservoirs in the proto-solar system from which the planets accreted. The bulk oxygen content is crucial, given the potential importance of volatile trapping within water ice in for formation of protoplanets in the outer solar system. Microwave spectroscopy, coupled with in situ sampling, offer some hope of determining these values in the four giant planets. 


\section{Giant Icy Moons}

In this section, we describe our current knowledge of the surface and subsurface characteristics of most of these objects, thus providing a general overview of the context (Fig. 2). Then, a second part will focus on the largest moons for which a thick liquid layer is suspected below the icy crust (Europa, Ganymede, and Callisto at Jupiter; Titan and Enceladus at Saturn). Those are the objects strongly motivating future space missions, partly because of their high astrobiological interest. The deep layers of these moons, and more specifically the main characteristics of the liquid layers, will be discussed.

\subsection{Surface and Space Environments}

The goal of this section is to describe surface compositions and volatile contents in the environments of Jupiter and Saturn for which most of the data have been acquired, but also to a lesser degree around Uranus and Neptune. A special focus is first given on water ice, which is by far the most abundant volatile compound making up the composition of the outer planets' moons. The nature of the water ice phases (amorphous versus crystalline) is discussed using the space-based hyperspectral evidences. Secondly, we will review nonwater-ice materials that have been detected on the surfaces of the icy moons. Finally, a third section will describe our current understanding of the composition of the atmospheres that surround the major moons.

\subsubsection{Water Signatures on Solid Surfaces}

Water ice, which formally is a mineral, shows diagnostic spectral signatures in the infrared range. Overall, the reflectance spectrum of water ice is temperature-dependent, and very sensitive to the average size of the grains composing the surface regolith (Clark 1981). The spectrum of water ice is dominated by a strong absorption band centered at about $3 \mu \mathrm{m}$ $\left(3250 \mathrm{~cm}^{-1}\right)$, resulting from the spectral counterparts of two fundamental vibration modes of the water molecule $\left(\mathrm{H}_{2} \mathrm{O}\right): v_{1}$ symmetric stretch, occurring at $2.941 \mu \mathrm{m}$ or $3400 \mathrm{~cm}^{-1}$, and $\nu_{3}$ asymmetric stretch, occurring at $3.105 \mu \mathrm{m}$ or $3221 \mathrm{~cm}^{-1}$, while the fundamental bending mode $v_{2}$ is responsible for a less intense absorption located just beyond $6 \mu \mathrm{m}$ $\left(1660 \mathrm{~cm}^{-1}\right)$. Combinations and overtones of these fundamental modes are represented by absorption bands falling in the near-infrared domain, centered at about 1.04, 1.25, 1.50, and $2.02 \mu \mathrm{m}$, and whose strength increases with increasing wavelength (Fig. 3, bottom).

Amorphous and crystalline ices are spectrally distinct, and as such they can be identified by comparing their spectra. Amorphous ice forms generally at temperatures lower than $\sim 130 \mathrm{~K}$. It can also be formed from crystalline ice by radiation of high-energy particles, such as ultraviolet photons, helium ions and high-energy electrons. Conversely, crystalline ice can be formed from amorphous ice by heating above 110-120 K (e.g. Newman et al. 2008). In crystalline water ice, one additional band is found at $1.65 \mu \mathrm{m}\left(6061 \mathrm{~cm}^{-1}\right)$, on the edge of the 1.5- $\mu \mathrm{m}$ band (Fink and Larson 1975). This feature is temperature-sensitive, such that it is deeper for colder crystalline ice. In spectroscopic measurements, this makes it a suitable marker of ice temperature, although this signature is also strongly affected by the grain size (Clark 1981). A reflectance peak at $3.1 \mu \mathrm{m}$ comes from the Fresnel reflection off the facets of the water ice grains in the surface. For this reason, the strength and shape of the $3.1 \mu \mathrm{m}$ peak does not vary significantly with grain size, as long as the grains are larger than a few microns in size. Amorphous ice can be qualitatively identified from a generally broad and featureless $3.1 \mu \mathrm{m}$ reflection peak, compared to a more pronounced and three-peaked 


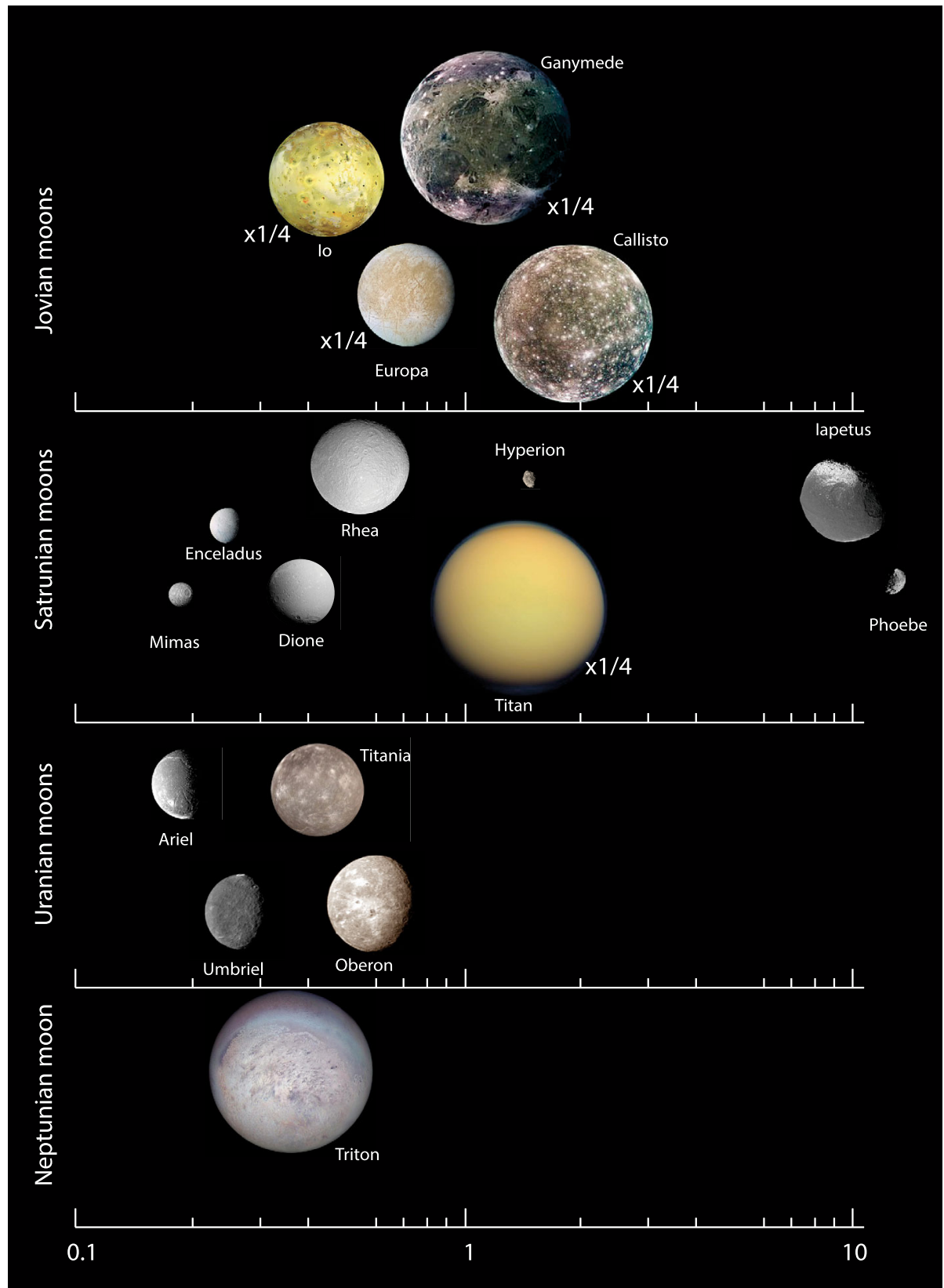

Fig. 2 Moons of the outer solar systems which are discussed in this paper. Except for Io, all these moons possess a significant amount of water and also volatiles and organics (see text for details). The horizontal axis indicates their distance to the planet (in million kilometers). All moons are at scale, except for the four giant moons of Jupiter, and for Titan at Saturn, which are scaled to 1/4 of their real size relative to the other moons 
structure in the crystalline spectrum (Grundy and Schmitt 1998; Hansen and McCord 2004; Mastrapa et al. 2009).

Long-period, Centaur, and Jupiter Family comets at heliocentric distances beyond $\sim 6 \mathrm{AU}$ are expected to host substantial amounts of amorphous ice. As for the icy moons, the distribution of crystalline vs. amorphous water ice on the surfaces is mapped by using a combination of some spectral markers diagnostic of ice crystallinity: the strength of the (temperature-sensitive) $1.65-\mu \mathrm{m}$ band, the position of the minimum of the $1.5-\mu \mathrm{m}$ and 2.0- $\mu \mathrm{m}$ bands, and the intensity and shape of the 3.1- $\mu \mathrm{m}$ Fresnel reflectance peak (e.g., Mastrapa et al. 2009). In general, amorphous ice spectra are characterized by wide and relatively unstructured bands. Upon crystallization, ice bands shift to longer wavelengths, become narrower, increase in intensity, and generally reveal new structures (Schmitt et al. 1998; Cruikshank et al. 1998a, 1998b, 2000a, 2000b; Hansen and McCord 2004; Clark et al. 2012). Such maps may reveal longitudinal variations due to weathering effects as well as latitudinal gradients due to radiation and local conditions (surface albedo). The average grain sizes can be inferred from the spectral continuum profile in the range between 3 and $4 \mu \mathrm{m}$, with finer grains showing a higher reflectance peak in this region and vice versa.

Based on hyperspectral data returned by the Near Infrared Mapping Spectrometer (NIMS) on-board the Galileo spacecraft, operating in the range 0.7-5.2 $\mu \mathrm{m}$ (Carlson et al. 1992), the icy moons of Jupiter mostly show evidence of crystalline ice at the surface, with some exceptions. On Callisto, NIMS spectra revealed that ice appears crystalline everywhere (where it is abundant enough to see), as expected from the average temperature of the satellite and from the low radiolytic fluxes. On Ganymede, sputtered ice particles easily reach the cold polar regions along Ganymede's magnetic field lines and form a thin layer of frost; the surface boundary between the open and closed field lines is clearly visible in color images (Khurana et al. 2007), with material mantling the polar regions that shows a higher albedo. In addition, the interaction with the Jovian magnetosphere was found to cause spectral differences with respect to the crystallinity and particle size of $\mathrm{H}_{2} \mathrm{O}$ ice between the equatorial and the polar regions of Ganymede, with a general trend from more crystalline ice at low latitudes to more amorphous ice in the polar regions (Hansen and McCord 2000b, 2004; Stephan 2006; Stephan et al. 2009). This is caused by the configuration of Ganymede's own magnetic field that protects at least partly surface regions between $\pm 40^{\circ}$ latitude from impacting magnetospheric particles (however, local conditions produce large variations in this trend). On Europa, where cold temperatures and high radiation fluxes would favor amorphous ice, the resemblance to the crystalline ice model is poor (Hansen and McCord 2000a), and although the amorphous ice model is not an exact fit, the spectrum is very sensitive to the exact morphological state of the amorphous deposit. Spectral signatures reveal an average fine grain size on Europa $(<100 \mu \mathrm{m})$, while larger and rough grains are found on Callisto (Roush et al. 1990; Calvin and Clark 1991). In all satellites of the Jovian system, finer grains tend to concentrate in the leading hemispheres, which is probably the result of micrometeorite gardening, combined with sputtering effects on the trailing side (Calvin and Clark 1993).

Hyperspectral images returned by the Visual and Infrared Mapping Spectrometer (VIMS) on-board the Cassini orbiter, covering the overall spectral range 0.35-5.1 $\mu \mathrm{m}$ (Brown et al. 2004), enabled a thorough comparative analysis of Saturn's icy moons. Disk-integrated data revealed that crystalline water ice is also dominant on those surfaces (Filacchione et al. 2010). On Enceladus, whose very high albedo drives low daytime surface temperatures, spatially-resolved maps show water ice to be mostly crystalline, with a higher degree of crystallinity at the "tiger-stripe" cracks and a larger amorphous signature between these stripes (Newman et al. 2008). These results suggest an intriguing atmospheric environment over 
the south pole where amorphous ice is produced either through intense radiative bombardment, flash-freezing of cryovolcanic liquid, or rapid condensation of water vapor particles on icy microspherules or on the surface of Enceladus (Newman et al. 2008). Furthermore, by considering six spectral indices diagnostic of sub-micron particles, those terrains showing the deepest water ice absorption bands were found to correlate with the lowest amount of sub-micron particles (Scipioni et al. 2017). For a surface depleted in visually dark, nonice contaminants, like the average surface of Enceladus, this leads to the interpretation that the variation measured in the depth of the water ice absorption bands is directly related to a variation in grain size, rather than to a compositional gradient (Scipioni et al. 2017). On Rhea, both amorphous and crystalline ice are present in different amounts across the craters. While neither pure amorphous nor pure crystalline $\mathrm{H}_{2} \mathrm{O}$ ice are widespread, crystalline ice is predominant in the center of the craters (Dalle Ore et al. 2015). A phase change from amorphous to crystalline may also occur as a result of localized heating of the ice by impacts of meteoroids in some circumstances (Stewart et al. 2008), and probably by micrometeoroids, which would explain the crystalline signature at $1.65 \mu \mathrm{m}$ on most outer Solar System bodies (Dalle Ore et al. 2015). Typical regolith grain sizes are smaller than on Jupiter's icy Galilean satellites, ranging between $38 \mu \mathrm{m}$ (Rhea) and $69 \mu \mathrm{m}$ (Tethys) (Filacchione et al. 2012).

\subsubsection{Nature and Distribution of Non-Water-Ice Surface Materials}

The nature and distribution of non-water-ice materials, and their relation with water ice, is a crucial point to understand the origin and evolution of the surfaces of the icy satellites, as surface material can be a link to the interior of the moons and provides constraints about the environment in which these bodies were formed and currently exist.

Distribution of Oxides In the icy Galilean satellites around Jupiter, the strength of the $\mathrm{CO}_{2}$ signature increases with increasing radial distance, moving away from Europa to Callisto. On Callisto, $\mathrm{CO}_{2}$ is more abundant on the trailing hemisphere, and in the interior, the rim and the ejecta of the major icy impact basins and many impact craters, with the youngest craters showing the largest abundance of this compound (Hibbitts et al. 2002). Since the impactor bodies cannot be the source of $\mathrm{CO}_{2}$, as this compound would rapidly sublimate at the temperature typical of the satellite's subsolar point $(\sim 170 \mathrm{~K})$, some kind of trapping structures (e.g., ice clathrates, physisorption) is invoked as a stable underground reservoir of $\mathrm{CO}_{2}$. On Ganymede, $\mathrm{CO}_{2}$ appears to be mostly correlated with the moderately hydrated non-ice material primarily associated with the dark regiones (Hibbitts et al. 2009) and, unlike Callisto, there does not appear to be any leading/trailing hemisphere asymmetry in the distribution of $\mathrm{CO}_{2}$ nor do impact craters tend to be $\mathrm{CO}_{2}$ rich (Hibbitts et al. 2003), while $\mathrm{CO}_{2}$ is occasionally enriched in terrain containing larger grained ice in comparison with adjacent terrain of similar morphology and ice abundance. On Europa, the $\mathrm{CO}_{2}$ band is present in the equatorial region of the leading hemisphere but is absent on the trailing hemisphere (Smythe et al. 1998; McCord et al. 1998a; Hansen and McCord 2009). Since impacts and meteoritic fall are greatest on the leading hemisphere, Europa's $\mathrm{CO}_{2}$ may be radiolytically produced in dark carbonaceous meteoritic deposits.

Ganymede also shows evidences of oxygen species, particularly solid $\mathrm{O}_{2}$ (Spencer et al. 1995) and $\mathrm{O}_{3}$ (Noll et al. 1996), in its trailing hemisphere, consistent with the preferential orientation of that side of the satellite with Jupiter's magnetosphere. Both of these species appear to be trapped within the ice matrix, and probably originate from ionic bombardment of the icy surface. Ultraviolet (UV) measurements of Ganymede carried out by the Galileo 
spacecraft confirmed the presence of ozone, whose abundance varies with latitude, with the strongest absorption measured at higher latitudes (Hendrix et al. 1999). This was interpreted as being the result of plasma bombardment creating $\mathrm{O}_{3}$ in the ice matrix and photodissociation destroying it, on a continual basis.

Io's colorful appearance is the result of various materials produced by its extensive volcanism. Io contains little to no water, though small pockets of water ice or hydrated minerals have been tentatively identified (Douté et al. 2004). Io's surface is largely dominated by sulphur species: in particular, $\mathrm{SO}_{2}$ frost is omnipresent (e.g. Douté et al. 2001), but there is also evidence for $\mathrm{S}_{2}, \mathrm{SO}, \mathrm{SO}_{2}$ gas and $\mathrm{NaCl}$ erupted from plumes (Lopes and Spencer 2007), as well as indications of Fe-bearing salts, silicates (feldspars and pyroxenes) consistent with high-temperature lava flows, $\mathrm{FeS}_{2}$, and iron sulphide minerals. Concerning $\mathrm{SO}_{2}$, it is also distributed on Callisto where it is generally mottled, with some areas of high concentrations correlated with ice-rich impact craters (Hibbitts et al. 2000). Large-scale patterns include the depletion of $\mathrm{SO}_{2}$ in the polar regions; and a depletion of $\mathrm{SO}_{2}$ on the trailing side relative to the leading side is observed. High-resolution data from Galileo/UVS (Hord et al. 1992) allowed the $\mathrm{SO}_{2}$ absorption to be mapped out across the surface of Europa (Hendrix et al. 1998, 2011), with a peak concentration near the apex of the trailing hemisphere. NIMSmeasured $\mathrm{SO}_{2}$ shows that it is not strongly correlated with $\mathrm{CO}_{2}$ but has a similar sparse distribution (Hansen and McCord 2008).

Volatiles such as $\mathrm{CO}_{2}$ were also found to be common on Saturn's larger icy satellites like Dione (Clark et al. 2008; Cruikshank et al. 2010; Stephan et al. 2010), Iapetus (Buratti et al. 2005), Hyperion (Cruikshank et al. 2008) and Phoebe (Clark et al. 2005), while it is less abundant on Rhea (Clark et al. 2008; Cruikshank et al. 2010; Stephan et al. 2012). Results from VIMS actually show that $\mathrm{CO}_{2}$ 's most diagnostic feature in the 3-5 $\mu \mathrm{m}$ range is slightly shifted to shorter wavelengths and broadened on Iapetus, Hyperion, and Dione compared to Phoebe (Cruikshank et al. 2010; Filacchione et al. 2010), thus revealing a different nature of the environment in which the $\mathrm{CO}_{2}$ molecule is trapped (Chaban et al. 2007). In the Saturnian system, $\mathrm{CO}_{2}$ is usually associated with dark material, suggesting that it is related to the interaction with incoming energetic particles from Saturn's magnetosphere or UV radiation and the emplacement or the formation of the rocky non-ice material (Stephan et al. 2010, 2012; Scipioni et al. 2013, 2014). However, Phoebe's $\mathrm{CO}_{2}$ is native to the body as part of the initial inventory of condensates and now exposed on the surface, strongly mixed with water ice and hydrocarbons (Coradini et al. 2008).

The albedos of the major Uranian moons, considerably lower than those of Saturn's moons (except Phoebe and the dark hemisphere of Iapetus), reveal that water ice is mixed in varying proportions to non-ice, visually dark and spectrally bland, material that is possibly carbonaceous in origin (Brown and Cruikshank 1983). $\mathrm{CO}_{2}$ has been detected from telescopic observations on Ariel, Umbriel and Titania, but has not been detected on the farthest regular Uranian satellite, Oberon (Grundy 2006). The detected $\mathrm{CO}_{2}$ ice appears to be concentrated on the trailing hemispheres of these satellites, and it decreases in abundance with increasing semi-major axis (Grundy 2006), as opposed to what is observed in the Saturnian system. The surface of Neptune's largest moon Triton is composed of a range of volatile-rich ices, including $\mathrm{N}_{2}, \mathrm{H}_{2} \mathrm{O}$ and $\mathrm{CO}_{2}$ (Quirico et al. 1999; Cruikshank et al. 2000b).

Nature and Distribution of Hydrated Minerals Hydrated, non-ice materials were identified by Galileo/NIMS on the icy Galilean moons Europa and Ganymede. These materials are concentrated in the visually dark regions of these satellites, showing low reflectance at wavelengths shortward of $3 \mu \mathrm{m}$ plus subdued and distorted water ice bands at 1.5 and $2.0 \mu \mathrm{m}$, as well as relatively high reflectance in the $3-5 \mu \mathrm{m}$ range compared to water ice 
Fig. 3 Galileo Near-Infrared Mapping Spectrometer (NIMS) spectra of Europa. The icy plains (middle) and dark, non-icy terrain (top) display absorption features arising from vibrational modes of the water molecule. The spectrum of crystalline water ice at $100 \mathrm{~K}$ (bottom) is shown for comparison. Note the appearance of the broad absorption features centered at 1.5 and $2.0 \mu \mathrm{m}$, and how their shapes differ in the Europa spectra. The asymmetric, distorted shapes seen in the less-icy terrain are attributed to a bound state such as water of hydration (from: Dalton et al. 2005)

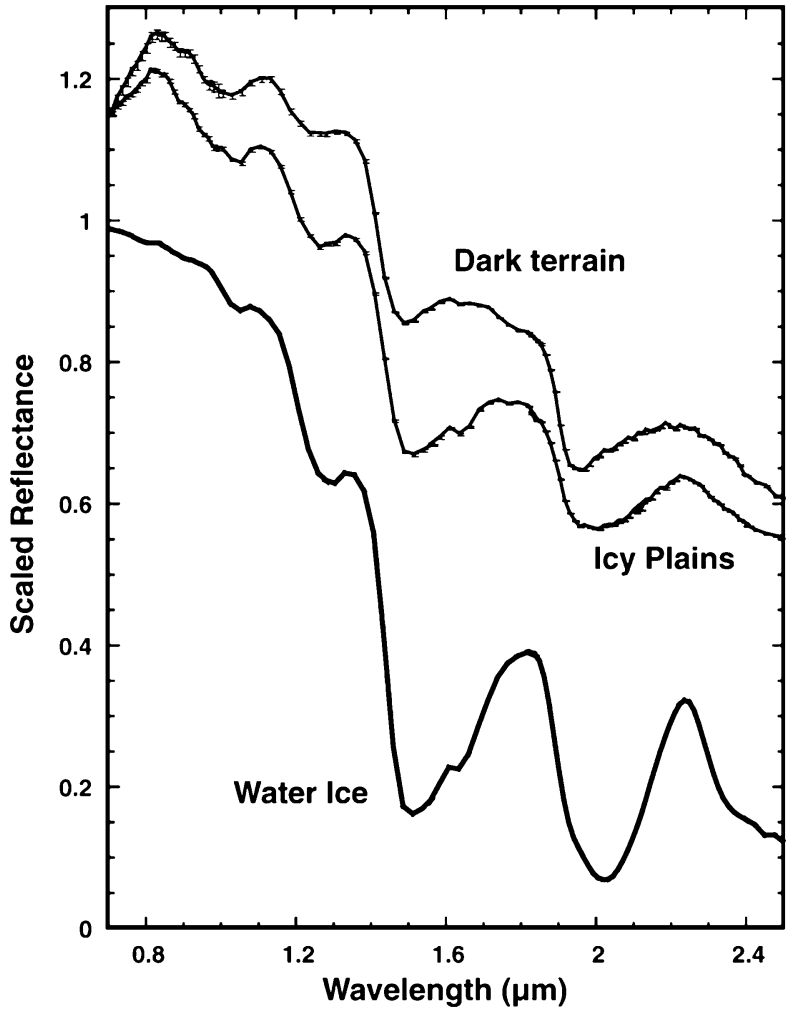

(McCord et al. 1998b, 1999) (Fig. 3). Compositional end-members invoked for this non-ice material are hydrated salt minerals enriched in $\mathrm{Mg}$ and $\mathrm{Na}$, which may form from the crystallization of brines erupted from the subsurface-these compounds were actually expected by the formation models of Europa (McCord et al. 1998b, 1999, 2001, 2002; Dalton 2003; Dalton et al. 2005; Dalton et al. 2012)—and hydrated sulphuric acid, which may result from the radiolysis of water and of the sulphur-bearing species, or from the decomposition of sulphate salts (Carlson et al. 1999). Recently, an alternative based on Mg-bearing chlorinated salts has been proposed for Europa (Ligier et al. 2016). Similar to water ice, the reflectance spectrum of hydrated salt minerals is very sensitive to grain size and temperature (e.g., Dalton 2003; Dalton et al. 2005; De Angelis et al. 2017).

Spectra of Europa's non-ice material are actually best matched by mixtures of these two compositional classes, i.e. sulphuric acid hydrate together with hydrated salts (McCord et al. 2001, 2002; Dalton 2007). One possible mechanism is that $\mathrm{Na}$ associated with some salts could be easily sputtered away and abundant $\mathrm{H}^{+}$could take its place, forming sulphuric acid (McCord et al. 2001, 2002). On Europa, the abundance of sulphuric acid hydrate is linked to the magnetospheric charged particle energy flux, and does not seem to strongly correlate with the underlying geologic strata. This could result from radiolytic processing of implanted sulphur from Io, or of sulphur emplaced as part of the surface deposits that came from the interior (Shirley et al. 2010; Dalton et al. 2013). The signature of the hydrated material, or the dark terrain, also varies according to geologic unit types (Shirley et al. 2010; Dalton et al. 2012). In particular, the hydrate absorption, perhaps salts and reformed sulphuric acid, are closely associated with at least some lineaments and is especially concen- 
trated near their centers, suggesting a source associated with the lineament formation mechanism (McCord et al. 2010; Dalton et al. 2012). The impact of particles trapped in Jupiter's magnetic field is very different on Ganymede than on Europa and no association with the Ganymede impact pattern is seen. Another possible source could be from exogenic material falling onto Ganymede's surface, but again the distribution only at some dark regions that are clearly exogenic from geologic arguments seems to argue for an exogenic origin.

The surfaces of Callisto and Ganymede also bear evidence of the presence of organic compounds, possibly tholins, i.e. nitrogen-rich organics that are found in cometary nuclei or that form by the action of charged particles impacting the icy surfaces (McCord et al. 1997, 1998a). The abundance of these compounds is tightly related to dark material, being weaker on Ganymede than on Callisto.

In the Saturn system, there is a trend of decreasing water ice band strength as a function of the radial distance, marking an increasing contribution of non-ice contaminants moving away from the planet (Filacchione et al. 2007). Using diagnostic spectral indices, such as spectral slopes and band depths in the VIS-IR range, a comparative analysis of the icy Saturnian moons carried out on the basis of disk-integrated VIMS data revealed striking differences among ring regions and satellites, ranging from Enceladus and Calypso's bluish surfaces, which appear very bright, water-ice-rich, and almost uncontaminated, to the more distant Hyperion, Iapetus, and Phoebe where metals, organics, and carbon dioxide are mixed within water ice at hemispheric scale, resulting in overall lower albedos and-for Hyperion and the leading side of Iapetus_-redder spectra (Cruikshank et al. 2008; Tosi et al. 2010; Filacchione et al. 2010, 2012, 2013). Phoebe appears to be compositionally different from the intermediate-sized regular satellites of Saturn: its organic-rich composition as revealed by VIMS is unlike any surface yet observed in the inner Solar System, which supports the hypothesis that this satellite is coated by material of cometary or outer Solar System origin (Clark et al. 2005), ultimately confirming that it is a captured body (Johnson and Lunine 2005).

Finally, organic material has been detected in Saturn's ring particles. In general, ring particles appear to have peculiar properties, being the reddest objects in Saturn's system at visible wavelengths while maintaining sharp and intense water ice bands in the infrared range (Nicholson et al. 2008; Filacchione et al. 2012; Cuzzi et al. 2010; Hedman et al. 2013). This spectral behavior is compatible with crystalline water ice polluted by chromophores, e.g., organic material resulting from the irradiation of simple hydrocarbons (Johnson et al. 1983; Moore et al. 1983), nanophase iron or hematite (Clark et al. 2012, 2008), tholins in intimate mixing (Ciarniello et al. 2011), amorphous silicates (Poulet et al. 2003), carbonaceous particles (Cuzzi and Estrada 1998), or different combinations of these end-members.

\subsubsection{Atmospheres, Plumes and Jets in the Icy Moons of the Outer Planets}

All of the four Galilean satellites are known to have thin atmospheres (McGrath et al. 2004), whose properties are indicative of surface processes and composition. Io's $\mathrm{SO}_{2}$ atmosphere, arising from volcanic emissions and sublimation, is the densest with an estimated surface pressure of the order of $10^{-9}$ bar. Additional species, including $\mathrm{SO}, \mathrm{S}_{2}, \mathrm{O}, \mathrm{S}, \mathrm{Na}, \mathrm{K}$ and $\mathrm{Cl}$, have also been detected as constituents of Io's atmosphere. The presence of an $\mathrm{O}_{2}$ atmosphere at Europa has been inferred from measurements of UV emissions (Hall et al. 1995, 1998); $\mathrm{Na}$ and $\mathrm{K}$ have also been measured at Europa, in ground-based observations (Brown and Hill 1996; Brown 2001). Ganymede also has a thin $\mathrm{O}_{2}$ atmosphere, inferred from measurements of UV emissions, and a hydrogen exosphere, measured by Galileo/UVS 
in a limb scan (Barth et al. 1997). The exosphere of Callisto is dominated by $\mathrm{O}_{2}$, detected on the basis of Hubble Space Telescope (HST) spectra acquired in the far-ultraviolet (Cunningham et al. 2015). Callisto's atmosphere is collisional and is the fourth-densest satellite atmosphere in the Solar System, in addition to being the second-densest $\mathrm{O}_{2}$-rich collisional atmosphere in the Solar System, after Earth (ibid). The Galileo/NIMS spectrometer also discovered exospheric $\mathrm{CO}_{2}$ (Carlson 1999). In 2012, direct images obtained by HST in the far-UV enabled the first detection of water vapor plumes at Europa, possibly as high as 200 $\mathrm{km}$ (Roth et al. 2014). Absorption from gas or aerosols beyond the Europa limb was then confirmed on the basis of further far-UV images obtained by HST in 2014, as the satellite transited the smooth face of Jupiter (Sparks et al. 2016).

Jets of water ice from surface fractures near the south pole of Saturn's icy moon Enceladus produce a plume of gas and particles (Dougherty et al. 2006; Waite et al. 2006). Enceladus' gaseous plumes were sampled with the Cassini Saturn Orbiter's INMS (Waite et al. 2004) during several fly-bys of the moon and found to be composed predominantly of $\mathrm{H}_{2} \mathrm{O}$ vapor, with $\mathrm{CO}_{2}$ as the second most abundant species. Ammonia is also present in the plumes, along with various organic compounds, deuterium and ${ }^{40} \mathrm{Ar}$ (Waite et al. 2009). The Cosmic Dust Analyzer (CDA) on-board the Cassini Orbiter (Srama 2004) also discovered salts in E-ring particles derived from the plume. These E-ring particles were determined to be rich in sodium salts $(\sim 0.5-2 \%$ by mass $)$ and were said to arise only if Enceladus' plume originates from liquid water that had been in contact with rocky interior material (Postberg et al. 2009).

Titan is the only satellite in the Solar System to have a thick, dense atmosphere, mainly composed of $\mathrm{N}_{2}(98.4 \%), \mathrm{CH}_{4}(1.4 \%)$ and $\mathrm{H}_{2}(0.1 \%)$, with trace amounts of several other gases and hydrocarbons. Thanks to the Cassini-Huygens mission, the atmosphere of Titan has now been extensively studied (e.g. Niemann et al. 2010) and presents a very complex chemistry. In particular, Titan's lower atmosphere harbor organic aerosols (tholins) presumed to be formed from simple molecules, such as methane and nitrogen. Cassini particle spectrometers indicate that a complex ion-neutral chemistry in Titan's upper atmosphere near $1000 \mathrm{~km}$ plays a major role in the formation of progressively more complex hydrocarbon molecules, from benzene to polycyclic aromatic hydrocarbons (PAH) and ultimately to aerosol particles with $\sim 260$-nm radii (Waite et al. 2007).

In situ measurements obtained by the Cassini spacecraft at Saturn's moons Dione and Rhea revealed tenuous atmospheres composed of $\mathrm{O}_{2}$ and $\mathrm{CO}_{2}$ (Teolis et al. 2010; Teolis and Waite 2016). Cassini magnetic field and plasma observations from the close flybys of Saturn's icy moon Dione are also consistent with the presence of an exosphere rich in molecular oxygen ions $\left(\mathrm{O}_{2}^{+}\right)$(Simon et al. 2011; Tokar et al. 2012). The atmospheres of Dione and Rhea appear to be sustained by chemical decomposition of the surface water ice under irradiation from Saturn's magnetospheric plasma. The presence of $\mathrm{CO}_{2}$ suggests radiolysis reactions between surface oxidants and organics or sputtering and/or outgassing of $\mathrm{CO}_{2}$ endogenic to ice. $\mathrm{O}_{2}: \mathrm{CO}_{2}$ source rates at Dione (Rhea) are estimated to be $\sim 30: 1$ (2:1) relative, consistent with a stoichiometric bulk composition below the lag layer of $0.01(0.13)$ $\mathrm{C}$ atoms per water molecule, deriving from endogenic constituents, implanted micrometeoritic organics, and (especially at Dione) exogenous $\mathrm{H}_{2} \mathrm{O}$ delivery by E-ring grains (Teolis and Waite 2016).

Finally, plumes consisting of nitrogen gas and dust were observed emanating from the southern polar cap of Triton (Soderblom et al. 1990). It is also known that Triton possesses a tenuous global atmosphere composed mainly of molecular nitrogen, with a trace of methane near the surface (Broadfoot et al. 1989). 


\subsection{Deep Water Reservoirs in Giant Satellites}

In this section, we summarize the main evidence from the Galileo era that have been driving the science community towards the hypothesis that liquid layers are indeed present in several moons. For each moon that may possess an ocean, it reviews briefly what are the main characteristics from the subsurface to the core, with a special focus on the evidence that suggests the presence of deep liquid reservoirs below these crusts. The main characteristics of the crustal material inferred from the surface observations are briefly summarized. Europa at Jupiter and Enceladus at Saturn will be looked at in a comparative manner, and a similar approach will be used for discussing Callisto and Ganymede at Jupiter, and Titan at Saturn. In the second subsection, the main characteristics of these liquid reservoirs both in term of composition and dynamics will be looked at.

\subsubsection{How Deep Are the Oceans?}

Recent or present geological activity, involving water rising from the shallow subsurface, has been demonstrated on Enceladus and Europa. Enceladus is indeed famous because of the geysers activity on its south pole. Globally, Enceladus exhibits four broad terrain regions: cratered plains, western hemisphere fractured plains, eastern hemisphere fractured plains, and the only active area called South Polar Terrain (Spencer et al. 2009). This is the area where Cassini found the tiger stripes, linear cracks about $130 \mathrm{~km}$ in length and $2 \mathrm{~km}$ wide. The tiger stripes are extremely active, producing a huge thermal emission, and outstanding plume jets, which composition and nature have been extensively studied (see Sect. 3.1.3). Even if these geysers cannot be considered as a proof of the existence of a global ocean, they represent at least a clear indication of the fact that a liquid reservoir must be present below the icy crust. The exact nature of this reservoir (locally distributed below the south pole, or global ocean) has been debated for about 10 years and is not yet entirely solved. First interpretations of the Cassini data favored an icy crust of about $40 \mathrm{~km}$ thick at the South pole and a thickening of the shell when approaching the equator of the moon. Global ocean or deep reservoir at the South pole were both envisaged. But in 2015, libration measurements were obtained, and favor the existence of a global ocean (Cadek et al. 2016). Cadek et al. (2016) also suggest that the ice shell might be much thinner than previously thought $(<20 \mathrm{~km})$, a prediction difficult to reconcile with other geophysical data. Nonetheless, the authors propose an original concept which might be consistent with all available data. Enceladus could be layered as follows: a rocky core, an internal global ocean approximately $45 \mathrm{~km}$ deep, and a $20-\mathrm{km}$ thick ice shell averaged. The South pole should be thinner $(<5 \mathrm{~km}$ thick). In this model, the ocean beneath the ice is about $40 \%$ of the total volume of the moon, and in direct contact with the silicate core.

Europa's surface is characterized by an extremely young surface, as indicated by its very low impact crater density (only 16 craters with diameters of 3-27 km). Europa's surface can be subdivided into bright (bluish color) plains, featuring numerous parallel ridges in a wide range of orientations, and darker, brownish mottled terrains. Linear ridges are the most widespread landforms on Europa; the most common of which are double ridges, consisting of a pair of ridges with a medial trough. The latter are thought to have originated through a variety of mechanisms, including, e.g., tectonism, cryovolcanism, or diapirism, and require either the presence of liquid water in the shallow subsurface, or warm mobile ice underlain by a deep ocean (Greeley et al. 2004; Schmidt et al. 2011). More recently, evidence for convergent areas were also suggested, thus providing for the first time clear indications of the possible recycling of the crust through a process similar to plate tectonics on Earth (Kattenhorn and Prockter 2014). The evidence for a large 
( 200-km high) plume of water vapor provided by Hubble Space Telescope (HST) imaging of far-UV emissions from excited $\mathrm{H}$ and $\mathrm{O}$ water dissociation products (Roth et al. 2014; Sparks et al. 2016) suggest that active processes may currently be at work at Europa. In any case, the surface of Europa suggests recently active processes operating in the ice shell. Although relatively little is known about the internal structure, most models include an outer ice shell underlain by liquid water, a silicate mantle, and an iron-rich core (Schubert et al. 2009). The existence of the liquid ocean, globally distributed below the icy crust, is still not proven without any doubt, but it corresponds to the best model that explains all geophysical data of the Galileo mission. In particular, it is now considered to be the only way to produce a shallow electrical current interacting with the jovian field to produce the induced magnetic field that has been detected by Galileo (Kivelson et al. 2009). The thickness of the crust is still highly uncertain. Two categories of models have been proposed suggesting either a very shallow liquid ocean only a few kilometers deep or an ocean trapped below a few tens of kms (Billings and Kattenhorn 2005). In Nimmo et al. (2003), a synthesis and analysis of all the data available at that time were provided suggesting a rather strong preference for the second hypothesis with an averaged value of the crustal thickness around $25 \mathrm{~km}$.

Ganymede, the largest moon of the Jovian system, presents a mix of very old and younger terrains revealing a geological record of several billions of years, ancient impact basins and fresh craters, and landscapes formed via tectonism, possibly icy volcanism, and slow-rate degradation by space weathering. In the Jovian system, Ganymede holds a key position because it features old, densely-cratered terrain, similar to most of Callisto's surface, but also widespread tectonically resurfaced regions, resembling a large part of Europa's surface. Ganymede does not show any evidence of activity at present, or even recent features. In its past history, the locally restricted depressions called paterae are the only features, which, to our knowledge, are seriously interpreted as being formed from cryovolcanic processes. At least one of them was interpreted as an icy flow that produced smooth bright lanes within the grooved terrain (Giese et al. 1998). This suggests that cryovolcanism in combination with tectonic processes may have played a role in the past for the formation of the bright terrain. But geologic evidences do not support the existence of shallow liquid reservoirs, similar to what has been suspected on Europa (Schmidt et al. 2011). On the other hand, strong evidences of the presence of deep and global liquid layers below the icy crust have been provided by the Galileo mission, with the detection of an induced magnetic field generated in response to the time-variable rotating magnetosphere of Jupiter (Kivelson et al. 2002; Kivelson 2004). The available data are still inconclusive but it is worth noting that the presence of the ocean is in agreement with geophysical models which predict that tidal dissipation and radiogenic energy keep the water liquid (e.g., Spohn and Schubert 2003; Hussmann et al. 2006), and also with the very recent observation from the Hubble Space Telescope of the oscillations of Ganymede's auroral ovals (Saur et al. 2015). Ganymede possesses a high-level of differentiation, meaning that there must be a clear separation between an iron metallic core (which existence is proven by the intrinsic magnetic field of the moon), a silicate mantle, and a hydrosphere about $500 \mathrm{~km}$ thick. Grasset et al. (2013) showed that all models converge towards an ocean thickness that varies from $50 \mathrm{~km}$ at 130 $\mathrm{km}$ in depth in the case of pure water, up to $230 \mathrm{~km}$ at about $60 \mathrm{~km}$ depth if ammonia were added to the liquid.

Callisto is characterized by globally abundant dark, densely cratered plains, indicating a very old surface, almost unchanged for billions of years. Callisto should be considered as a window into the early history of the outer solar system. Its surface is dominated by various impact features, similar in morphology to those observed on Ganymede, and by 
landforms indicative of intense surface erosion and degradation. Callisto is also characterized by a dark blanket covering most of its surface. This is due to a process called sublimation degradation, most certainly triggered by the presence of $\mathrm{CO}_{2}$, that caused the degradation of bright high-standing terrain (e.g., crater rims) and the formation of this globally abundant dark, smooth blanket. Unfortunately, the time-scale of this dark lag formation is not known (Moore et al. 2004). Crater forms suggest that rheological properties and subsurface layering may be similar to Ganymede, but degradation states and ages of the palimpsests (craters with a specific morphology) infer different rates of change of these properties with time. Tectonism is not widespread but a few systems (furrows and lineaments) indicate that a small geologic activity occurred in the past (Moore et al. 2004; Prockter et al. 2010). Galileo data suggest that Callisto is a partially differentiated body. The very old surface of Callisto indicating an absence of activity for billions of years, may imply a total lack of dynamics in its deep interior. But this is not consistent with Galileo detection of an induced magnetic field that could possibly be due to the presence of a large liquid reservoir below the outer icy shell, or an induced magnetosphere produced by a substantial and conducting ionosphere. This moon being not in the Laplace resonance (resonance that locks the three inner moons into a state where tidal heating is intense, especially for Io and Europa), there is no other sources of heat than the radiogenic heating from the silicates. That fact, plus the fact that the moon may not be fully differentiated indicate that Callisto remains enigmatic. The discovery by Galileo of the induced magnetic field is still hard to reconcile with the geophysical state of the moon. In any case, the putative ocean should be located under a thick crust, most certainly thicker than all other moons since this crust has been there almost from the origins of Callisto.

Titan is very similar to Ganymede in terms of size and bulk composition. Its internal structure is still mostly unknown but the moment of inertia indicates a moderately differentiated body not possessing an inner metallic core but an hydrous silicate core, overlain by an hydrosphere which must be about the same thickness as on Ganymede. Cassini gravity science observations also revealed that Titan hosts a deep ocean (Iess et al. 2012). But Titan is unique in the solar system because of its thick atmosphere, and because of the exchange processes between this atmosphere, the surface, and possibly the icy crust. The massive atmosphere is mainly composed of $\mathrm{N}_{2}(\sim 98 \%)$ and $\mathrm{CH}_{4}(\sim 2 \%)$ (e.g. Griffith et al. 2013). It is beyond the scope of this paper to elaborate on the extreme complexity of Titan's atmospheric dynamics, but it is worth noting that Titan is an excellent laboratory for exploring the diversity of volatiles that can be found with the water-rich material of the outer solar system, and may still be present inside the large satellites. First, the low ${ }^{36} \mathrm{Ar} / \mathrm{N}_{2}$ abundance measured by the Gas Chromatograph Mass Spectrometer onboard Huygens indicates that nitrogen has been brought on Titan in the form of N-bearing compounds, most likely $\mathrm{NH}_{3}$ (Atreya et al. 2009). The ${ }^{14} \mathrm{~N} /{ }^{15} \mathrm{~N}$ ratio measured in $\mathrm{N}_{2}$ is very close to the value recently inferred in $\mathrm{NH}_{2}$ radicals in comets (Rousselot et al. 2014; Shinnaka et al. 2014), suggesting a similar origin. Huygens also detected a small amount of $\mathrm{CO}_{2}$ in the upper atmosphere, that could have originated from a recombination of $\mathrm{CO}$ and $\mathrm{OH}^{-}$(Hörst et al. 2008). $\mathrm{CO}_{2}$ has also been observed at different locations on Titan's surface from IR spectral absorption (McCord et al. 2008), and possibly in the upper crust as suggested by the $\mathrm{CO}_{2}$ evaporation subsequent to the landing of the Huygens probe (Niemann et al. 2010). Mandt et al. (2014) suggested that Titan's building blocks might have a composition close to comets. Unfortunately, even if comets were representative of the initial composition of planetesimals in the vicinity of Saturn's forming region, the fraction of volatile compounds that was lost or modified during the accretion processes is still unknown (e.g. Estrada et al. 2009). Nonetheless, it is worth noting that the major species which can 


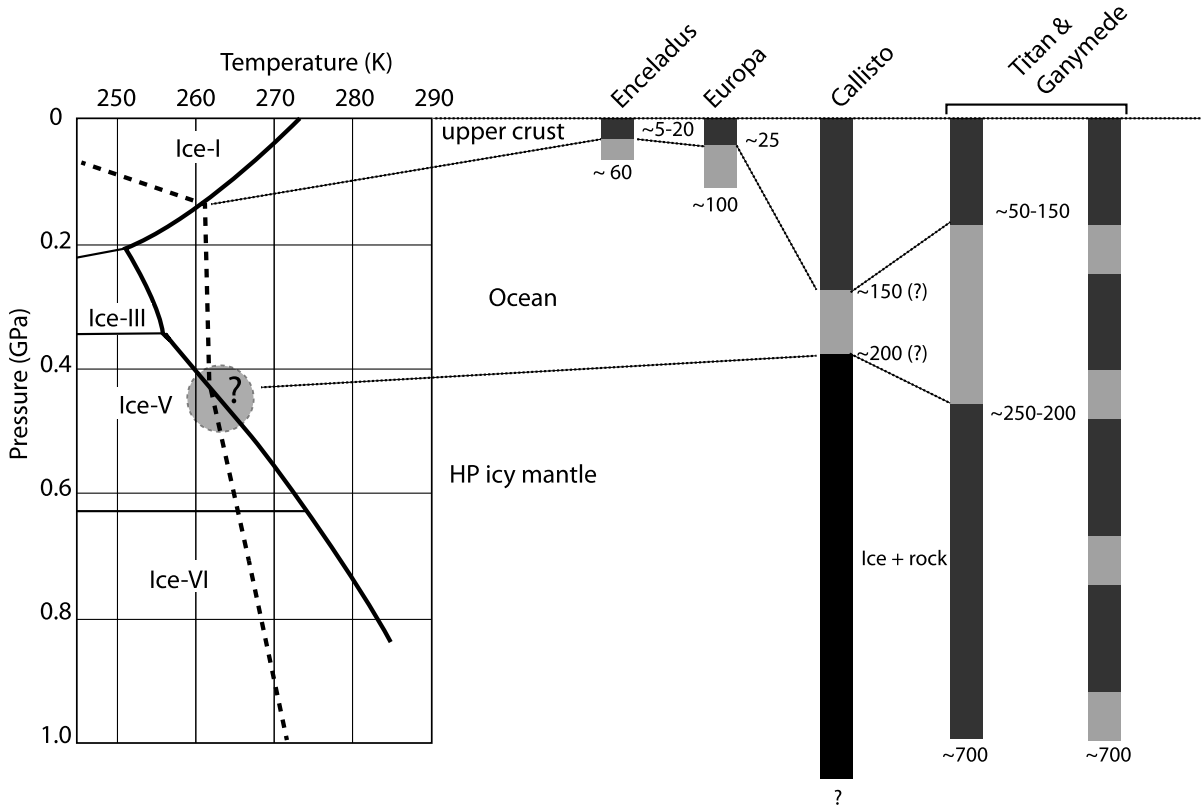

Fig. 4 Left: Phase diagram of the pure water system in the pressure-temperature range relevant for icy moons (bold lines: melting curves; thin lines: solid-solid transitions; dashed line: a possible thermal profile-see text for details) Right: hydrosphere layering of the giant moons. Positions of the interfaces are indicative of the pressure range only, but their precise location depends on temperature profiles within the hydrospheres and on the composition of the icy material. Numbers are depth ranges in km (not at scale) (Enceladus; Cadek et al. 2016; Europa: Nimmo et al. 2003; Callisto: Kuskov and Kronrod 2005; Titan \& Ganymede-left: Tobie et al. 2005; Hussmann et al. 2015; right: from Vance et al. 2014)

be easily mixed with liquid water in small amounts, or combined with water ice through hydration or clathration processes must have been incorporated into Titan's interior in the primordial stages (Tobie et al. 2006). Further observations are needed to better describe the exchange processes within this moon from its primordial stage to its present state, which calls for future missions. As for Ganymede, there is no way to derive a precise estimate of the crustal thickness and of the size of the liquid reservoir. Too many uncertainties remain, especially when considering the possible compositions of the liquid and the icy crust, key parameters in determining the position of the liquid-ice interfaces. Nonetheless, it is commonly agreed that Titan's liquid reservoir should be about $100 \mathrm{~km}$ deep (e.g., Tobie et al. 2005).

\subsubsection{Structure and Dynamics of Deep Hydrospheres}

Deep liquid layers trapped below a thick icy crust seem to be a common feature in the giant moons of the outer solar system. At this point, it is necessary to make a distinction between the two, and possibly more, different natures of oceans that could exist. At first order this is related to the amount of water available, i.e., to the pressure range that is imposed on it. Coming back to the pressure-temperature phase diagram of water (Fig. 4) first established by Bridgman (1937), and just slightly modified since then (see Choukroun and Grasset 2010 for a review), one can see that the pressure domain is a key issue for understanding the nature of deep reservoirs. Without considering any chemical complexity (pure water only), many 
ice polymorphs already exist in the moderate pressure range 0-2 GPa, relevant to icy moons. But the main characteristics of the diagram that is worth noticing is that the melting curve of the low-pressure ice Ih decreases with pressure, which is related thermodynamically to the fact that ice Ih is less dense than its liquid. The other ices, existing above $0.209 \mathrm{GPa}$ in the case of the pure water system, are denser than the liquid and display melting curves with positive slopes. This is why two kinds of liquid reservoirs can already be imagined. When the hydrosphere is thin (such as for Enceladus or Europa), the liquid reservoir is most certainly trapped in between an icy crust and a silicate mantle, because the pressure at the bottom of the hydrosphere is below the critical pressure above which high pressure polymorphs exist. In that case, the liquid ocean is most certainly in contact with the rocky core, thus providing interesting possibilities for chemical exchanges through geothermal processes, extremely relevant for the astrobiological potential of these moons. Hydrothermal vents are indeed extremely favorable places for complex chemical evolution, thanks to the heterogeneous processes occurring between the hot gases and liquid and solid phases (Raulin 2005). On the other hand, for the largest moons and most probably all water-rich exoplanets with cold surfaces, the only way for a liquid layer to exist is to be trapped between the icy crust and a lower layer of high-pressure (HP) ices. Internal pressures are sufficient to allow for the formation of high-pressure ice phases below the ocean, thus splitting the hydrosphere into a high-pressure ice layer consisting of various water-rich ices denser than liquid water, the subsurface water ocean, and an ice-I layer forming the outer crust of the satellites.

The thermal structure of the hydrospheres is commonly described from top to bottom using the scaling laws derived from numerical modelling of convective processes within thick icy mantles. In fact, they were first modeled in the early 80 s after the Voyager missions returned evidence for endogenic activity (Cassen et al. 1982; Friedson and Stevenson 1983) and the field has been expanding ever since. Following the pioneering models of Lewis (1971) or Consolmagno and Lewis (1978), early thermal evolutions for large icy moons (Reynolds and Cassen 1979; Kirk and Stevenson 1987) either did not consider internal oceans or put enormous emphasis on the freezing of transient oceans in terms of convective and tectonic implications - see for example the work of Kirk and Stevenson (1987) in the case of Ganymede. More recent studies have taken into account the possibility/likelihood of present-day internal oceans above high-pressure icy mantles (e.g. Showman et al. 1997; Tobie et al. 2005; Hussmann et al. 2015). The inner structures displayed in Fig. 4 were calculated assuming hydrostatic and thermal equilibrium and matching available moments of inertia and mass balance constraints. All these models assume thermal equilibrium, i.e., that the heat produced both from radiogenic decay in the silicates and tidal heating is entirely transferred through the thick high-pressure layer, if present, the ocean, and finally the upper icy mantle. The interface depths vary between studies because of the chosen physical parameters and scaling laws. For example, the ocean thickness depends on assumptions made on thermal gradients in the icy layer. Since thermal profiles cannot be measured, our understanding of the deep interior structure remains model-dependent.

Features commonly accepted for the two giant moons Ganymede and Titan include: a high-pressure ice mantle about $400 \mathrm{~km}$ thick; a deep ocean about $250 \mathrm{~km}$ thick; an icy upper layer ranging from a few tens to about one hundred km thick. For Callisto, the upper icy layer and the ocean may have the same characteristics than for the two other large satellites, but the limited differentiation inferred from available gravity data implies that there is no highpressure icy mantle. The interior model of Europa displayed in Fig. 4 has an upper crust a few tens of $\mathrm{km}$ thick in average (Nimmo et al. 2003). An alternative scenario suggests that the icy crust could not be more than a few km thick (see Billings and Kattenhorn 2005). Future missions such as the Europa Orbiter mission planned by NASA for the next decade 
will help retire this uncertainty via a combination of ground penetrating radar and gravity science. If Europa's crust is thinner than a few kilometers, the ESA JUICE mission will also be capable to detect the ocean below (Grasset et al. 2013). Finally, the interior structure of Enceladus displayed in Fig. 4 is based on the latest model of Cadek et al. (2016).

Icy and liquid layers are composed of pure water. Icy compositions found in cometary material and on moon surfaces indicate that hydrospheres must host a complex chemistry. Unfortunately, the nature of this complex chemistry is still mostly unknown. As already mentioned in Sect. 3.1, many volatiles and organic compounds have been detected on icy surfaces and may originate from the deep interior of these bodies. Ammonia is also suspected, especially on Titan. These compounds add complexity to the simple model proposed above, for two mains reasons: volatiles reduce the melting temperature of the ice (especially ammonia); clathrate and hydrate structures may form, hence increasing the fraction of solid materials present in the hydrosphere. Regarding the first point, the minimum temperature allowing for deep oceans to exist is about $250 \mathrm{~K}$ in case of pure water ice, but could be substantially smaller with the presence of antifreeze compounds such as ammonia (down to $180 \mathrm{~K}$ ). This property influences significantly the size of the liquid reservoir for a given thermal profile. As for the second point, recent studies of Ganymede (Vance and Brown 2013; Vance et al. 2014) illustrate the importance of hydrated materials. An original internal structure has been proposed based on the sandwiched ocean scenario, but with more than one liquid layer because of the density contrasts between the liquid, the pure ices, and the hydrated and/or clathrated ices through depth. It is rather hard to reconcile this alternative model with dynamical constraints based on thermochemical models of the moons, but it is a very attractive and interesting alternative that shows clearly how imprecise is our current understanding of the deep interior of the moons.

In Fig. 4, the question mark at the interface at the bottom of the ocean illustrates a singularity, which to our knowledge, has not yet been resolved for the largest moons and possibly water-rich exoplanets. While the thermal profile postulates a simple temperature increase in the HP ice mantle, either corresponding to an isentropic increase (ascribed to extremely efficient convection) or to an increase in conductive transfer, the actual temperature profile most probably differs from this simple picture. As the HP ice mantle thickness probably amounts to several hundreds of kilometers, and knowing the orders of magnitudes for the viscosity values of high pressure polymorphs, vigorous subsolidus convective instabilities must occur. For this reason, a conductive profile is not realistic. Subsolidus thermal convection is, notwithstanding, much less efficient than in the ocean so that an isentropic profile will not extend up to the liquid ocean/HP mantle interface and a cold boundary layer will build up. Estimates (Choblet and Tobie 2015) show that the slope of this cold thermal boundary layer should exceed that of the ice melting curve which suggests that the upper portion of the HP mantle is partially molten. This state is not stable over geologic time scales since the entire system is out of thermal equilibrium. Preliminary simulations also show that significant melting should occur in the warmest regions of the HP mantle with two major consequences: (1) melt production and melt delivery to the ocean above is a major heat sink; (2) in most models, melt is predicted to occur in the deeper-most region of the HP ice layer in contact with the rock core. These calculations suggests that chemical/mass transfer is probably not prohibited by the HP mantle and point to the need for a better assessment of the dynamics of the HP mantle in the context of an evolving ocean/HP mantle interface. As noted above, planetary models lack both a proper description of the high-pressure ice mantle dynamics and an adequate description of the liquid-solid interface. While the configuration of an evolving melting/crystallizing front is encountered in many different settings in Earth and planetary sciences (e.g. magma oceans evolution, growth of a solid inner core within metallic 
cores), it has mostly been addressed with a focus on convection in the low viscosity liquid. A proper description at longer time-scales, involving solid-state convection, remains to be developed.

\section{Transneptunian Objects and Ceres}

This section focuses on large planetesimals found beyond Neptune's orbit. The past decade has seen the discovery of a large number of large bodies in the Kuiper Belt at a large pace (see Brown 2013, for a review). Larger objects are suspected beyond the detection capability of the current telescopes via their influence on the dynamical behavior of the Kuiper Belt and scattered disk (e.g., Batygin and Brown 2016). These objects are expected to be dominated in volume by water ice and other volatiles such as methane, methanol, nitrogen, etc. McKinnon et al. (2016) pointed out that the activity inferred at Sputnik Planitia based on nitrogen ice is naturally expected at other Kuiper belt objects. More generally, Schenk and Nimmo (2016) demonstrated that similar geological processes are found across the outer solar system driven by whichever volatiles condensed in abundance, or even second-phase impurities as a function of the distance to the Sun. This section also includes Ceres, a dwarf planet located in the main belt of asteroids, but believed to have acquired its volatiles from outer solar system planetesimals (De Sanctis et al. 2015).

\subsection{Dwarf Planet Ceres}

Evidence for a large fraction of volatiles, about 50 vol.\%, was inferred from Ceres' density of about $2.16 \mathrm{~g} / \mathrm{cm}^{3}$ (McCord and Sotin 2005). Water may be partitioned between phyllosilicates and other hydrated materials (e.g., salts) and ice. Early models of Ceres' internal evolution suggested that Ceres would have differentiated a thick ice shell (McCord and Sotin 2005; Castillo-Rogez and McCord 2010). However, Ceres' geomorphology turned out to tell a different story (Fig. 5a). While its shape is globally relaxed to hydrostatic equilibrium (Park et al. 2016), Ceres has preserved topographic features of scale $130 \mathrm{~km}$ and smaller, including unrelaxed craters that require a mechanically strong shell (Bland et al. 2017). Bland et al. (2017) inferred that the shell should contain at least 50vol.\% of nonice, material to explain the observed geomorphology, which may point to an enrichment in silicates and hydrated salts, possibly also clathrates (Fu et al. 2017; Castillo-Rogez et al. 2017). While water ice might be a minor component of Ceres' shell, its presence is expressed in a variety of features: surface exposure (Combe et al. 2016, 2017), ground ice flows (Schmidt et al. 2017), permanently shadowed regions (Schorghofer et al. 2016). The gamma ray and neutron detector (GRaND) on-board the Dawn mission also detected a significant increase in near-surface $(1 \mathrm{~m})$ ice at latitudes greater than $45 \mathrm{deg}$. (Prettyman et al. 2017) consistently with the distribution of ground ice features (Schmidt et al. 2017). Indirect evidence for volatiles in Ceres' shell is expressed via large edifices such as Ahuna mons and the bright deposits in Occator's crater, whose emplacement was facilitated by the presence of liquid. Ahuna mons is a 4-km mean height mountain that requires the presence of a few percent melt, likely brines expected at depth by thermal models (Ruesch et al. 2016; Castillo-Rogez and McCord 2010). The Occator bright spots are dominated by sodium carbonates and ammonium salts (carbonates or chlorides, De Sanctis et al. 2016). The concentration of these species is explained by the creation of a local reservoir of melt upon impact-induced heat (Bowling et al. 2016). In this context the deposits are evaporites that concentrated as water vaporized from Ceres' airless surface. 


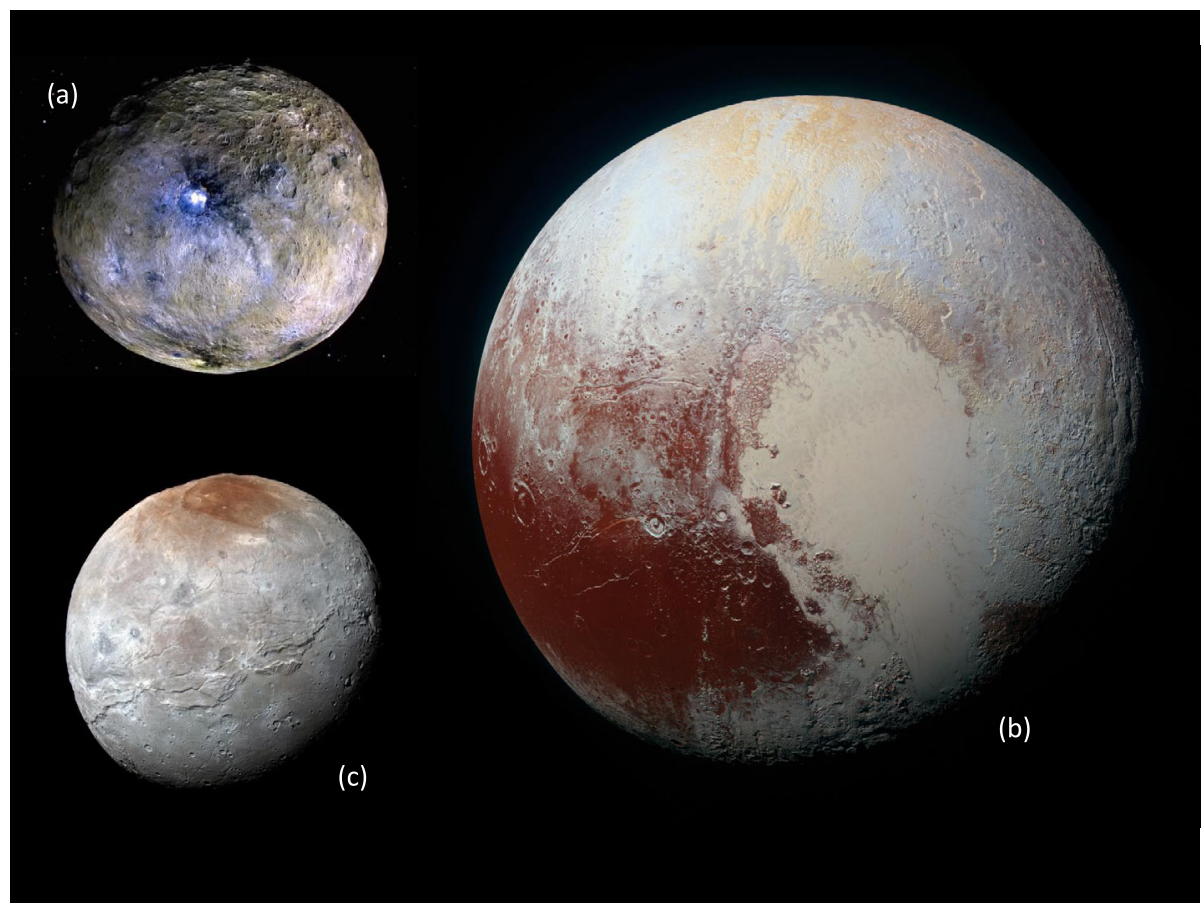

Fig. 5 Enhanced color images of (a) Ceres as seen by the Dawn mission (Credit NASA/JPL/DLR); (b) Pluton and (c) Charon as seen by the New Horizons mission (Credit: NASA/SwRI/APL). Color variations across the surface of these bodies reveal lithological variations that may be a combination of composition and physical processing (e.g., fresh vs. altered material). The prominent bright spot in Ceres' Occator crater has been characterized as mixture of salts but the very dark ejecta surrounding that crater remain to be interpreted. Reddish colors of Charon's and Pluto's surfaces reveal exotic ices and processed organics, in particular tholins

Besides water, Ceres accreted non-negligible amounts of ammonia and $\mathrm{CO} / \mathrm{CO}_{2}$. This is evidenced by the widespread occurrence of ammoniated materials and carbonates across Ceres' surface detected from ground-based observations (King et al. 1992; Rivkin et al. 2006) and confirmed by the Dawn mission (De Sanctis et al. 2015). Ammonia may have been accreted in the form of ammonia hydrates that turned into ammonium in solution during a phase when Ceres held a deep ocean (Castillo-Rogez et al. 2017). An alternative is that ammonia was accreted in the form of organics present in the early solar nebula and desorbed as a result of thermal metamorphism (McSween et al. 2017).

Several other main belt objects share similar evidence for the accretion of volatiles found in abundance in outer solar system planetesimals: the large 10 Hygiea and the smaller $(<200 \mathrm{~km})$ asteroids part of the Europa group of asteroids that display a $3.15 \mu \mathrm{m}$ absorption band associated with ammonia compounds by Takir et al. (2015). An origin of these objects in the outer solar system is likely and supports recent solar system dynamical models that simulate a large migration events from planetesimals in the 8 to $15 \mathrm{AU}$ region in the early solar system history (e.g., Walsh et al. 2011). An alternative scenario is these asteroids formed in situ and accreted planetesimals migrated from the outer solar system (Mousis et al. 2008; Grazier et al. 2014). However, migration across the nebula gap opened by Jupiter would have led to the depletion of these planetesimals of their most volatile species (especially 
ammonia) (Turner et al. 2012). Hence Ceres and other large asteroids are likely to be related to outer solar system bodies, and potentially giant planet satellites.

\subsection{Pluto and Charon}

Pluto and its satellite system were revealed in great detail during the approach and flyby of the New Horizons mission, between January 2014 and July 2015 (Figs. 5b and 5c). New Horizons confirmed the abundance of water ice as well as other volatiles detected from ground-based and space telescopes (Cruikshank et al. 2015). The dwarf planet's surface also presents a variety of additional volatiles, including nitrogen $\left(\mathrm{N}_{2}\right)$, methane $\left(\mathrm{CH}_{4}\right)$, and carbon monoxide (CO) ices (Grundy et al. 2016). Pluto's landscape has been shaped by glacial flow where water plays the role of bedrock (Moore et al. 2016). It experienced major extensional tectonism and resurfacing, probably of cryovolcanic origin, nearly 4 billion years ago. It also shows evidence for cryovolcanism in the form of mounds and a possible supervolcano in the Southern hemisphere (http:/www.nasa.gov/feature/possible-icevolcano-on-pluto-has-the-wright-stuff).

The impact of volatile materials on Pluto's geology is expressed in the diversity of the landscapes found on this dwarf planet. Like Ceres and Mars, Pluto displays pitted terrains that express the loss of volatiles by sublimation and other volatile-driven processes. Furthermore, several features suggest that Pluto could be endogeneously active today. One is the presence of a large basin, called Sputnik Planitia, that was formed less than 10 My ago. Sputnik Planitia is dominated by nitrogen ice. McKinnon et al. (2016) suggested that the peculiar morphology of this region, which displays an assemblage of polygons, is evidence for sluggish convection (a special style of convection in which deep material is upwelled up to the surface) over a 1-km deep layer. Nimmo et al. (2016) pointed out that Sputnik Planitia had to reorient, which implies the presence of a subsurface ocean in Pluto, such as those already described in Sect. 3.2. Furthermore, Singer and Stern (2016) pointed out that Pluto's nitrogen atmosphere is not stable over long periods and cannot be replenished solely from cometary supply, hence it may come from Pluto's interior.

Less information is available for Pluto's satellites. However, water ice has been found to dominate their surfaces and geology (Weaver et al. 2016). Pluto's largest satellite, Charon is relatively simpler than Pluto and more akin to icy satellites in its geological properties. Grundy et al. (2016) found a surface dominated by water ice as well as ammonia in some fresh craters. Reddish material at its poles appears due to tholins. Large, hemispheric-wide scarps observed on Charon may be ascribed to stresses generated by the cooling of a global ocean.

\subsection{Transneptunian Objects (KBOs, Centaurs, Scattered Disk Objects)}

This subsection reviews the state of knowledge of icy composition found at transneptunian objects. These are known to be volatile rich based on their relatively low densities, in general lower than $2 \mathrm{~g} / \mathrm{cm}^{3}$. Ice has been commonly found on these bodies (see, e.g., Barucci et al. 2006, 2011; Schaller and Brown 2008). Brown et al. (2012) found that members in the Haumea family exhibit almost pure water ice surfaces, which they interpreted as evidence for the differentiation of a thick icy mantle in their parent. These authors also found a possible relationship between the occurrence of ice and ammonia and large KBOs that could point to recent or ongoing internal activity. Terai et al. (2016) showed that water ice on at least three large KBOs is a mixture of crystalline and amorphous phases. These authors suggested 


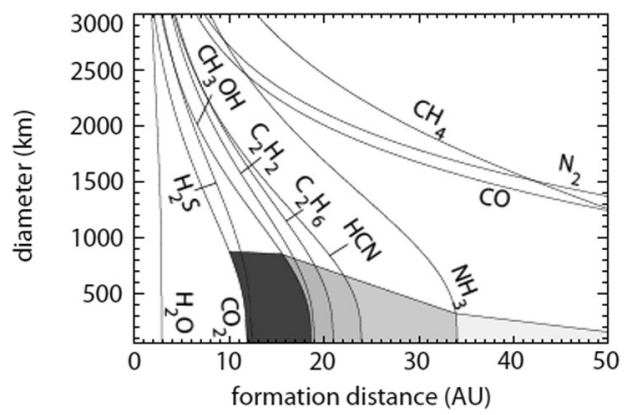

Fig. 6 Evaporation gradients in the early Solar system as a function of object size, which drives the evaporation timescale. The shaded regions show the formation regions of the three main surface types of KBOs: in the inner part of the primordial Kuiper Belt only water ice and carbon dioxide are stable over long timescales. In the middle part of the Kuiper Belt methanol is retained, in the outer part ammonia is preserved (from Brown et al. 2011, permission pending)

a possible correlation between the abundance of crystalline ice and the size of the objects, although more observations are required for robust linkage.

Kuiper Belt objects are frequently featureless (see for example Fornasier et al. 2009). However, ammonia, methane, and nitrogen have been frequently found on the surfaces of KBOs and TNOs, for example (50000) Quaoar, and the dwarf planet (13699) Eris (e.g., Merlin et al. 2009). Carry et al. (2011) confirmed the presence of ammonia at a level of a few percent on the surface of Orcus and further identified the presence of methane. Barucci et al. (2005) found a mixture of nitrogen, methane, and methanol ices, besides tholins. Methanol has been suggested to be responsible for the red colors of some Centaurs and KBOs believed to have formed beyond the methanol evaporation line (Brown et al. 2012). Specifically, the very red crustal material observed on some of these bodies has been suggested as the product of irradiation of a mixture of ice, carbon dioxide, and methanol (e.g., Brunetto et al. 2006). However, other explanations are possible, for example mixtures of organic tholins (e.g., Cruiskshank and Dalle Ore 2013).

Schaller and Brown (2007) showed that the escape of volatile compounds can explain the diversity in surface volatile content, and the frequent absence of volatile altogether, at these bodies. These authors also suggested that KBOs smaller than $1000 \mathrm{~km}$ are too small to retain volatiles on their surface for extended periods of time. They mapped out the stability of various volatiles as a function of distance to the Sun (Fig. 6). Ammonia is not expected to be stable over extended periods of time because it is rapidly destroyed by irradiation (e.g., Cooper et al. 2004), less than 0.1 Myr after Cook et al. (2007). Hence the occurrence of ammonia implies a relatively recent emplacement either via cryovolcanism acting on a regional scale or a large impact that excavated part of the deep interior. DeMeo et al. (2008) pointed out that the widespread presence of crystalline ice in the TNO population also suggests recent emplacement since ice tends to amorphize upon long exposure to space weathering.

Centaurs are very interesting objects in that some can be outgassing and reveal their primitive composition. Observations so far have detected ice and frozen methanol or light hydrocarbons, for example at 5145 Pholus (Cruiskshank et al. 1998). Carbon monoxide has also been found on a few Centaurs including 174P/Echelus and 2060 Chiron (e.g., Wierzchos et al. 2017). Many observations of Centaurs have revealed surface variations, which Merlin et al. (2005) attributed to impacts (e.g., Licandro and Pinilla-Alonso 2005) or as 
signature of activity (outgassing), as expected for these bodies when they start approaching their perihelia.

A major open question for that class of objects is whether some of their volatiles (e.g., methane, carbon monoxide and dioxide) were trapped as ice, in amorphous ice, and/or acquired via clathrate hydrates (e.g., McKinnon 2002). A similar question applies to comets. This could a priori be addressed by tracking the type of ice in Centaurs outgassed as a function of distance to the Sun (e.g., Leliwa-Kopystynski et al. 2013), since the release temperature would be different for these materials. However, modelling this is complicated while observations are scarce, so this questions remains open at present.

Abundant ice can be a vector of long-term activity in dwarf planets and other large TNOs provided that they also contain enough rock as a source of radioisotope-based heating. Hydrated silicates have been suggested at the surface of some of these bodies (e.g., Busarev et al. 2003) that would testify of a protracted phase of aqueous alteration involving at least partial melting of their interiors (Desch et al. 2009). The Plutino (90482) Orcus displays potential spectral evidence for ammonium (Delsanti et al. 2010), a marker of aqueous alteration, like in the case of Ceres (De Sanctis et al. 2015), although other types of nitrogen compounds can explain the Orcus spectra (e.g., $\mathrm{NH}_{3}$, Barucci et al. 2008). Our current understanding of the evolution of large icy satellites and Pluto is relevant to that class of bodies, but key unknowns are the conditions under which cryovolcanism would develop and the role of second-phase, low-eutectic species in promoting ice mobility, as explored by Kargel et al. (1991).

\section{Conclusion}

In this paper, a broad overview of the nature and distribution of water and volatiles in the outer solar system has been proposed. In the first part, the focus was placed on the giant and intermediary planets. The moons, and especially those in which a liquid ocean is expected, were described in the second part. Finally, a third section was dedicated to Ceres and the dwarf planets in the Kuiper Belt.

To summarize, Jupiter and Saturn most likely contain more volatiles than all the other objects in the solar system added together. But the volatile abundances remain poorly known as they are hidden away at great depths within the planets. On Uranus and Neptune, the amount of volatiles and ices is even more uncertain due to the lack of constraints. The exact nature and distribution of the elements in the deep interior of giant and intermediary planets are still highly unknown. In the gaseous atmospheres, volatile species are in trace quantities but they play a crucial role in shaping the dynamics, clouds, and general circulation of the atmospheres. On that specific matter, a detailed analysis of the current knowledge on the four planets has been proposed. Most of the water and volatiles not trapped in the planets are in the moons, and to a much lesser extent in their tenuous atmospheres (Titan excepted). The exact nature and distribution of volatiles and ices differ significantly from one planetary system to the other, and among moons within a system. But there is a general consensus that volatiles and possibly organic compounds can be found on all surfaces, even if it is sometimes marginal. For most of the moons, the relationships between the surface characteristics and the internal structure and dynamics is highly uncertain, except in a few cases where evidence for deep oceans yields insights into the coupling processes at work. Finally, the two best known examples of dwarf planet systems (Ceres, Pluto and its moons), and other KBOs have been addressed. It turns out that their bulk characteristics in terms of nature and distribution of water and ices at their surfaces do not differ significantly from the icy moons. 
Significant progress has been made these last two decades thanks to the two flagship missions Cassini-Huygens at Saturn, and Galileo at Jupiter. Additional datasets recently returned by New Horizons in the Pluto system, Dawn at Ceres, and also from ground-based observations at all wavelengths and for all planetary objects have provided a rather complete overview of the nature and the diversity of the water-rich bodies in the outer system. A next and significant step will be achieved by the Juno mission at Jupiter, especially in probing the deep atmosphere to better constrain the coupling processes from the atmosphere to the deep interior of Jupiter. After the completion of the Cassini mission in 2017, there will not be any further exploration of the icy moons for at least one decade. Then, the planned NASA mission to Europa and the ESA JUICE mission to Ganymede will provide new constraints on the Jovian system. These two missions will carry the instrumentation needed to accurately determine the nature and distribution of the volatile and organic compounds on the two moons, and to better characterize their hydrospheric structures and thermal states. In 2016 NASA and ESA kicked off a joint study about the exploration of the icy giant systems. These missions are envisioned for the 2020-2030 timeframe. Other advanced concepts for the exploration of the outer solar system are being devised, including a probe into Saturn's atmospheric composition (e.g., Mousis et al. 2016) and Titan Lake Lander (Mitri et al. 2014).

\section{References}

R.K. Achterberg, B.J. Conrath, P.J. Gierasch, Icarus 182, 169-180 (2006)

Y. Alibert, C. Mordasini, W. Benz, C. Winisdoerffer, Astron. Astrophys. 434, 343-353 (2005). doi:10.1051/ 0004-6361:20042032

S.K. Atreya, P.R. Mahaffy, H.B. Niemann, M.H. Wong, T.C. Owen, Planet. Space Sci. 51, 105-112 (2003)

S.K. Atreya, R.D. Lorenz, J.H. Waite, in Titan from Cassini-Huygens (2009), pp. 177-199

F. Bagenal, T.E. Dowling, W.B. McKinnon, Jupiter: The Planet, Satellites and Magnetosphere, 2nd edn. (Cambridge University Press, Cambridge, 2006)

K.H. Baines, R.W. Carlson, L.W. Kamp, Icarus 159, 74 (2002)

D. Banfield, P. Gierasch, M. Bell, E. Ustinov, A. Ingersoll, A. Vasavada, R. West, M. Belton, Icarus 135(1), 230-250 (1998)

C.A. Barth et al., Geophys. Res. Lett. 24, 2147-2150 (1997)

M.A. Barucci, D.P. Cruikshank, E. Dotto, F. Merlin, F. Poulet, C. Dalle Ore, S. Fornasier, C. de Bergh, Astron. Astrophys. 439, L1-L4 (2005)

M.A. Barucci, F. Merlin, E. Dotto, A. Doressoundiram, C. de Bergh, Observations of the TNO 55638 (2002 $\mathrm{VE}_{95}$ ) and analysis of the population's spectral properties. Astron. Astrophys. 455, 725-730 (2006)

M.A. Barucci, F. Merlin, A. Guilbert, C. de Bergh, A. Alvarez-Candal, O. Hainaut, A. Doressoundiram, C. Dumas, T. Owen, A. Coradini, Astron. Astrophys. 479, L13-L16 (2008)

M.A. Barucci, A. Alvarez-Candal, F. Merlin, I.N. Belskaya, C. de Bergh, D. Perna, F. DeMeo, S. Fornasier, Icarus 214, 297-307 (2011)

K. Batygin, M.E. Brown, Astron. J. 151(2), 22 (2016)

M. Beuthe, A. Rivoldini, A. Trinh, Geophys. Res. Lett. (2016). doi:10.1002/2016GL070650

S.E. Billings, S.A. Kattenhorn, Icarus 177, 397-412 (2005)

G.L. Bjoraker, M.H. Wong, I. de Pater, M. Adamkovics, Astrophys. J. 810, 122 (2015)

M.T. Bland, C.A. Raymond, R.R. Fu, P. Schenk, T. Kneissl, J.H. Pasckert, H. Hiesinger, F. Preusker, R. Park, S. Marchi, S. King, J.C. Castillo-Rogez, C.T. Russell, Nat. Geosci. 10, 338-343 (2017). doi:10.1038/ngeo2936

A.C. Boley, T. Hayfield, L. Mayer, R.H. Durisen, Icarus 207, 509-516 (2010). doi:10.1016/j.icarus. 2010.01.015

T. Bowling, F.J. Ciesla, S. Marchi, B.C. Johnson, T.M. Davison, J.C. Castillo-Rogez, M.C. De Sanctis, C.A. Raymond, C.T. Russell, Lunar and Planetary Science Conference (2016), p. 47

P.W. Bridgman, J. Chem. Phys. 5, 964 (1937)

F.H. Briggs, P.D. Sackett, Icarus 80, 77-103 (1989)

A.L. Broadfoot et al., Science 246, 1459-1466 (1989)

M.E. Brown, Icarus 151(2), 190-195 (2001)

M.E. Brown, Astrophys. J. Lett. 778(2), L34 (2013) 
R.H. Brown, D.P. Cruikshank, Icarus 55, 83-92 (1983)

M.E. Brown, R.E. Hill, Nature 380(6571), 229-231 (1996)

R.H. Brown et al., Space Sci. Rev. 115(1-4), 111-168 (2004)

M.E. Brown, E.L. Schaller, W.C. Fraser et al., Astrophys. J. Lett. 739, L60 (2011)

M.E. Brown, E.L. Schaller, W.C. Fraser, Astrophys. J. 143, 146 (2012)

R. Brunetto, M.A. Barucci, E. Dotto, G. Strazzulla, Ion irradiation of frozen methanol, methane, and benzene: linking to the colors of Centaurs and trans-Neptunian objects. Astrophys. J. 644, 646 (2006)

B.J. Buratti et al., Astrophys. J. 622(2), L149-L152 (2005)

V.V. Busarev, V.A. Dorofeeva, A.B. Makalkin, Earth Moon Planets 92, 345-357 (2003)

O. Cadek, G. Tobie, T. Van Hoolst, M. Masse, G. Choblet, L. Lefevre, G. Mitri, R.-M. Baland, M. Behounkova, O. Bourgeois, A. Trinh, Geophys. Res. Lett. (2016). doi:10.1002/2016GL068634

W.M. Calvin, R.N. Clark, Icarus 89, 305-317 (1991)

W.M. Calvin, R.N. Clark, Icarus 104(1), 69-78 (1993)

R.W. Carlson, Science 283(5403), 820 (1999)

R.W. Carlson et al., Space Sci. Rev. 60(1-4), 457-502 (1992)

R.W. Carlson et al., Science 286(5437), 97-99 (1999)

B. Carry, D. Hestroffer, F.E. DeMeo, A. Thirouin, J. Berthier, P. Lacerda, B. Sicardy, A. Doressoundiram, C. Dumas, D. Farrelly, T.G. Muller, Astron. Astrophys. 534, A115 (2011)

P.M. Cassen, S.J. Peale, R.T. Reynolds, in Satellites of Jupiter (University of Arizona Press, Tucson, 1982), pp. 93-128 (A83-16226 04-91)

J.C. Castillo-Rogez et al. Meteorit. Planet. Sci. (2017, submitted)

J.C. Castillo-Rogez, T.B. McCord, Icarus 205, 443-459 (2010). doi:10.1016/j.icarus.2009.04.008

T. Cavalié, R. Moreno, E. Lellouch, P. Hartogh, O. Venot, G.S. Orton, C. Jarchow, T. Encrenaz, F. Selsis, F. Hersant, L.N. Fletcher, Astron. Astrophys. 562, A33 (2014). doi:10.1051/0004-6361/201322297c

G.M. Chaban et al., Icarus 187(2), 592-599 (2007)

G. Choblet, G. Tobie, AGU abstract P23D-06. AGU Fall Meeting (2015)

M. Choukroun, O. Grasset, J. Chem. Phys. 133, 144502 (2010)

M. Ciarniello et al., Icarus 214(2), 541-555 (2011)

R.N. Clark, J. Geophys. Res. 86, 3087-3096 (1981)

R.N. Clark et al., Nature 435(7038), 66-69 (2005)

R.N. Clark et al., Icarus 193(2), 372-386 (2008)

R.N. Clark et al., Icarus 218(2), 831-860 (2012)

J.-Ph. Combe, T.B. McCord, F. Tosi, E. Ammannito, F. Carrozzo, M.-C. De Sanctis A. Raponi, S. Byrne, M.E. Landis, K. Hughson, C. Raymond, C. Russell, Science 353(6303), id.aaf3010 (2016)

J.-Ph. Combe, A. Raponi, F. Tosi, M.C. De Sanctis, F.G. Carrozzo, F. Zambon, E. Ammannito, K.H.G. Hughson, A. Nathues, M. Hoffmann, T. Platz, G. Thangjam, N. Schorghofer, S. Schröder, S. Byrne, M.E. Landis, O. Ruesch, T.B. McCord, K.E. Johnson, S.M. Singh, C.A. Raymond, C.T. Russell, Exposed $\mathrm{H}_{2} \mathrm{O}$-rich areas detected on Ceres with the Dawn Visible and InfraRed mapping spectrometer. Icarus (2017, submitted)

B.J. Conrath, P.J. Gierasch, S.S. Leroy, Icarus 83, 255-281 (1990)

G.J. Consolmagno, J.S. Lewis, Icarus 34, 280-293 (1978)

J.C. Cook, S.J. Desch, T.L. Roush, C.A. Trujillo, T.R. Geballe, Astrophys. J. 663, 1406 (2007)

J.F. Cooper, E.R. Christian, J.D. Richardson, C. Wang, in The First Decadal Review of the Edgeworth-Kuiper Belt (2004), pp. 261-277

A. Coradini et al., Identification of spectral units on Phoebe. Icarus 193(1), 233-251 (2008)

D.P. Cruikshank et al., Ices on the satellites of Jupiter, Saturn, and Uranus, in Solar System Ices, ed. by B. Schmitt, C. deBergh, M. Festou (Kluwer Academic, Dordrecht, 1998a), pp. 579-606

D.P. Cruikshank, T.L. Roush, T.C. Owen, E. Quirico, C. deBergh, The surface compositions of Triton, Pluto, and Charon, in Solar System Ices, ed. by B. Schmitt, C. deBergh, M. Festou (Kluwer Academic, Dordrecht, 1998b), pp. 655-684

D.P. Cruikshank et al., Surface composition of Hyperion. Nature 448(7149), 54-56 (2000a)

D.P. Cruikshank et al., Water ice on Triton. Icarus 147(1), 309-316 (2000b)

D.P. Cruikshank et al., Hydrocarbons on Saturn's satellites Iapetus and Phoebe. Icarus 193(2), 334-343 (2008)

D.P. Cruikshank et al., Carbon dioxide on the satellites of Saturn: results from the Cassini VIMS investigation and revisions to the VIMS wavelength scale. Icarus 206(2), 561-572 (2010)

D.P. Cruikshank, W.M. Grundy, F.E. DeMeo, M.W. Buie, R.P. Binzel, D.E. Jennings, C.B. Olkin, J.W. Parker, D.C. Reuter, J.R. Spencer, S.A. Stern, L.A. Young, H.A. Weaver, The surface compositions of Pluto and Charon. Icarus 246, 82-92 (2015)

D.P. Cruiskshank, C.M. Dalle Ore, Earth Moon Planets 92, 315-330 (2013) 
D.P. Cruiskshank, T.L. Roush, M.J. Bartholomew, T.R. Geballe, Y.J. Pendleton, S.M. White, J.F. Bell III, J.K. Davies, T.C. Owen, C. de Bergh, D.J. Tholen, Icarus 135, 389-407 (1998)

N.J. Cunningham, J.R. Spencer, P.D. Feldman, D.F. Strobel, K. France, S.N. Osterman, Icarus 254, 178-189 (2015)

J.N. Cuzzi, P.R. Estrada, Icarus 132(1), 1-35 (1998)

J.N. Cuzzi et al., An evolving view of Saturn's dynamic rings. Science 327(5972), 1470-1475 (2010)

C.M. Dalle Ore, D.P. Cruikshank, R.M.E. Mastrapa, E. Lewis, O.L. White, Impact craters: an ice study on Rhea. Icarus 261, 80-90 (2015)

J.B. Dalton, Astrobiology 3(4), 771-784 (2003)

J.B. Dalton, Geophys. Res. Lett. 34(21), L21205 (2007)

J.B. Dalton et al., Spectral comparison of heavily hydrated salts with disrupted terrains on Europa. Icarus 177(2), 472-490 (2005)

J.B. Dalton et al., Europa's icy bright plains and dark linea: exogenic and endogenic contributions to composition and surface properties. J. Geophys. Res. 117(E3), E03003 (2012)

J.B. Dalton et al., Exogenic controls on sulfuric acid hydrate production at the surface of Europa. Planet. Space Sci. 77, 45-63 (2013)

S. De Angelis et al., Temperature-dependent VNIR spectroscopy of hydrated Mg-sulfates. Icarus 281, 444458 (2017)

T. de Graauw, H. Feuchtgruber, B. Bezard, P. Drossart, T. Encrenaz, D.A. Beintema, M. Griffin, A. Heras, M. Kessler, K. Leech, E. Lellouch, P. Morris, P.R. Roelfsema, M. Roos-Serote, A. Salama, B. Vandenbussche, E.A. Valentijn, G.R. Davis, D.A. Naylor, Astron. Astrophys. 321, L13-L16 (1997)

I. de Pater, S.T. Massie, Icarus 62, 143-171 (1985)

I. de Pater, P.N. Romani, S.K. Atreya, Icarus 91, 220-233 (1991)

I. de Pater, L.A. Sromovsky, P.M. Fry, H.B. Hammel, C. Baranec, K.M. Sayanagi, Icarus 252, 121-128 (2015)

I. de Pater, R.J. Sault, B. Butler, D. DeBoer, M.H. Wong, Science 352, 1198-1201 (2016)

M.C. De Sanctis et al., Ammoniated phyllosilicates with a likely outer Solar System origin on (1) Ceres. Nature 528, 241-244 (2015)

M.C. De Sanctis, A. Raponi, E. Ammannito, M. Ciarniello, M.J. Toplis, H.Y. McSween, J.C. Castillo-Rogez, B.L. Ehlmann, F.G. Carrozzo, S. Marchi, F. Tosi, F. Zambon, F. Capaccioni, M.T. Capria, S. Fonte, M. Formisano, A. Frigeri, M. Giardino, A. Longobardo, G. Magni, E. Palomba, L.A. McFadden, C.M. Pieters, R. Jaumann, P. Schenk, R. Mugnuolo, C.A. Raymond, C.T. Russell, Nature 536, 54-57.12 (2016)

A.D. Del Genio, R.K. Achterberg, K.H. Baines, F.M. Flasar, P.L. Read, A. Sanchez-Lavega, A.P. Showman, in Saturn from Cassini-Huygens, vol. 6 (Springer, Berlin, 2009), pp. 113-159

A. Delsanti, F. Merlin, A. Guilbert-Lepoutre, J. Bauer, B. Yang, K.J. Meech, Astron. Astrophys. 520, A40 (2010)

F. DeMeo, M.A. Barucci, A. Alvarez-Candal, C. de Bergh, S. Fornasier, F. Merlin, D. Perna, I. Belskaya, Icy bodies of the solar system, in Proceedings of the 263th of the International Astronomical Union, ed. by J.A. Fernandez, D. Lazzaro, D. Prialnik, R. Schulz (2008), pp. 186-191

S.J. Desch, J.C. Cook, T.C. Doggett, S.B. Porter, Icarus 202, 694-714 (2009)

M.K. Dougherty et al., Identification of a dynamic atmosphere at Enceladus with the Cassini magnetometer. Science 311(5766), 1406-1409 (2006)

M.K. Dougherty, L.W. Esposito, S.M. Krimigis, in Saturn from Cassini-Huygens, ed. by M.K. Dougherty, L.W. Esposito, S.M. Krimigis (Springer, Berlin, 2009). doi:10.1007/978-1-4020-9217-6

S. Douté et al., Mapping $\mathrm{SO}_{2}$ frost on Io by the modeling of NIMS hyperspectral images. Icarus 149(1), 107-132 (2001)

S. Douté et al., Geology and activity around volcanoes on Io from the analysis of NIMS spectral images. Icarus 169(1), 175-196 (2004)

T. Encrenaz, E. Lellouch, P. Drossart, H. Feuchtgruber, G.S. Orton, S.K. Atreya, Astron. Astrophys. 413, L5-L9 (2004)

P.R. Estrada, I. Mosqueira, J.J. Lissauer, G. D’Angelo, D.P. Cruikshank, Formation of Jupiter and conditions for accretion of the Galilean satellites, in Europa, ed. by R.T. Pappalardo (University of Arizona Press, Tucson, 2009), pp. 27-58

H. Feuchtgruber, E. Lellouch, T. de Graauw, B. Bezard, T. Encrenaz, M. Griffin, Nature 389, 159-162 (1997)

H. Feuchtgruber, E. Lellouch, G. Orton, T. de Graauw, B. Vandenbussche, B. Swinyard, R. Moreno, C. Jarchow, F. Billebaud, T. Cavalie, S. Sidher, P. Hartogh, Astron. Astrophys. 551, A126 (2013)

G. Filacchione et al., Saturn's icy satellites investigated by Cassini-VIMS: I. Full-disk properties: 350-5100 $\mathrm{nm}$ reflectance spectra and phase curves. Icarus 186(1), 259-290 (2007)

G. Filacchione et al., Saturn's icy satellites investigated by Cassini-VIMS: II. Results at the end of nominal mission. Icarus 206(2), 507-523 (2010) 
G. Filacchione et al., Saturn's icy satellites and rings investigated by Cassini-VIMS: III-radial compositional variability. Icarus 220(2), 1064-1096 (2012)

G. Filacchione et al., The radial distribution of water ice and chromophores across Saturn's system. Astrophys. J. 766(2), 76 (2013)

U. Fink, H.P. Larson, Icarus 24, 411-420 (1975)

G. Fischer, W.S. Kurth, D.A. Gurnett, P. Zarka, U.A. Dyudina, A.P. Ingersoll, S.P. Ewald, C.C. Porco, A. Wesley, C. Go, M. Delcroix, Nature 475, 75-77 (2011)

L.N. Fletcher, G.S. Orton, N.A. Teanby, P.G.J. Irwin, G.L. Bjoraker, Icarus 199, 351-367 (2009)

L.N. Fletcher, P. Drossart, M. Burgdorf, G.S. Orton, T. Encrenaz, Astron. Astrophys. 514, A17+ (2010)

L.N. Fletcher, K.H. Baines, T.W. Momary, A.P. Showman, P.G.J. Irwin, G.S. Orton, M. Roos-Serote, C. Merlet, Icarus 214, 510-533 (2011a)

L.N. Fletcher, B.E. Hesman, P.G.J. Irwin, K.H. Baines, T.W. Momary, A. Sanchez-Lavega, F.M. Flasar, P.L. Read, G.S. Orton, A. Simon-Miller, R. Hueso, G.L. Bjoraker, A. Mamoutkine, T. del Rio-Gaztelurrutia, J.M. Gomez, B. Buratti, R.N. Clark, P.D. Nicholson, C. Sotin, Science 332, 1413-1417 (2011b)

L.N. Fletcher, B.E. Hesman, R.K. Achterberg, P.G.J. Irwin, G. Bjoraker, N. Gorius, J. Hurley, J. Sinclair, G.S. Orton, J. Legarreta, E. García-Melendo, A. Sańchez-Lavega, P.L. Read, A.A. Simon-Miller, F.M. Flasar, Icarus 221, 560-586 (2012)

L.N. Fletcher, T.K. Greathouse, G.S. Orton, P.G.J. Irwin, O. Mousis, J.A. Sin- clair, R.S. Giles, Icarus 238, 170-190 (2014)

S. Fornasier, M.A. Barucci, C. de Bergh, A. Alvarez-Candal, F. DeMeo, F. Merlin, D. Perna, A. Guilbert, A. Delsanti, E. Dotto, A. Doressoundiram, Astron. Astrophys. 508, 457-465 (2009)

A.D. Fortes, M. Choukroun, Space Sci. Rev. 153, 185-218 (2010). doi:10.1007/s11214-010-9633-3

T. Fouchet, E. Lellouch, B. Bezard, T. Encrenaz, P. Drossart, H. Feuchtgruber, T. de Graauw, Icarus 143, 223-243 (2000)

T. Fouchet, P.G.J. Irwin, P. Parrish, S.B. Calcutt, F.W. Taylor, C.A. Nixon, T. Owen, Icarus 172, 50-58 (2004)

A.J. Friedson, D.J. Stevenson, Icarus 56(1), 1-14 (1983)

R. Fu, E. Ermakov, S. Marchi, J.C. Castillo-Rogez, C.A. Raymond, B.H. Hager, M.T. Zuber, S.D. King, M.T. Bland, M.C. De Sanctis, F. Preusker, R.S. Park, C.T. Russell, Earth Planet. Sci. Lett. (2017, submitted)

P.J. Gierasch, A.P. Ingersoll, D. Banfield, S.P. Ewald, P. Helfenstein, A. Simon- Miller, A. Vasavada, H.H. Breneman, D.A. Senske (Galileo Imaging Team), Nature 403, 628-630 (2000)

B. Giese, J. Oberst, T. Roatsch, G. Neukum, J.W. Head, R.T. Pappalardo, Icarus 135, 303-316 (1998)

R.S. Giles, L.N. Fletcher, P.G.J. Irwin, Icarus 257, 457-470 (2015)

O. Grasset, E.J. Bunce, A. Coustenis, M.K. Dougherty, C. Erd, H. Hussmann, R. Jaumann, O. PrietoBallesteros, Astrobiology 13(10), 991-1004 (2013). doi:10.1089/ast.2013.1013

K.R. Grazier, J.C. Castillo-Rogez, P.W. Sharp, Icarus 232, 13-21 (2014)

R. Greeley, C.E. Chyba, J.W. Head, T.B. McCord, W. McKinnon, R.T. Pappalardo, P.H. Figueredo, Geology of Europa, in Jupiter. The Planet, Satellites and Magnetosphere, ed. by F. Bagenal, T.E. Dowling, W.B. McKinnon. Cambridge Planetary Science, vol. 1 (Cambridge University Press, Cambridge, 2004), pp. 329-362. ISBN 0-521-81808-7

C.A. Griffith, J.L. Mitchell, P. Lavvas, G. Tobie, Titan's evolving climate, in Comparative Climatology of the Terrestrial Planets, ed. by S. Mackwell, M. Bullock, J. Harder (University of Arizona Press, Tucson, 2013)

W.M. Grundy, Icarus 184(2), 543-555 (2006)

W.M. Grundy, B. Schmitt, The temperature-dependent near-infrared absorption spectrum of hexagonal $\mathrm{H}_{2} \mathrm{O}$ ice. J. Geophys. Res. 103(E11), 25809-25822 (1998)

W.M. Grundy, R.P. Binzel, B.J. Buratti, J.C. Cook, D.P. Cruikshank, C.M. Dalle Ore, A.M. Earle, K. Ennico, C.J.A. Howett, A.W. Lunsford, C.B. Olkin, A.H. Parker, S. Philippe, S. Protopapa, E. Quirico, D.C. Reuter, B. Schmitt, K.N. Singer, A.J. Verbiscer, R.A. Beyer, M.W. Buie, A.F. Cheng, D.E. Jennings, I.R. Linscott, J.W. Parker, P.M. Schenk, J.R. Spencer, J.A. Stansberry, S.A. Stern, H.B. Throop, C.C.C. Tsang, H.A. Weaver, G.E. Weigle, L.A. Young, Science 351, id.aad9189 (2016)

T. Guillot, Science 269, 1697-1699 (1995). doi:10.1126/science.7569896

T. Guillot, Annu. Rev. Earth Planet. Sci. 33, 493-530 (2005)

T. Guillot, D. Gautier, Giant planets, in Treatise on Geophysics, vol. 10, ed. by G. Schubert 2nd edn. (Elsevier, Oxford, 2015), pp. 529-557

T. Guillot, R. Hueso, Mon. Not. R. Astron. Soc. 367, L47-L51 (2006). doi:10.1111/j.1745-3933.2006. 00137.x

T. Guillot, D.J. Stevenson, W.B. Hubbard, D. Saumon, in Jupiter. The Planet, Satellites and Magnetosphere, ed. by F. Bagenal, T.E. Dowling, W.B. McKinnon (2004), pp. 35-57

D.T. Hall, D.F. Strobel, P.D. Feldman, M.A. McGrath, H.A. Weaver, Nature 373(6516), 677-679 (1995)

D.T. Hall, P.D. Feldman, M.A. McGrath, D.F. Strobel, Astrophys. J. 499(1), 475-481 (1998) 
G.B. Hansen, T.B. McCord, in 31st Annual Lunar and Planetary Science Conference, March 13-17, 2000, Houston, TX (2000a), abstract no. 1630

G.B. Hansen, T.B. McCord, Bull. Am. Astron. Soc. 32, 1068 (2000b)

G.B. Hansen, T.B. McCord, J. Geophys. Res. 109(E1), E01012 (2004)

G.B. Hansen, T.B. McCord, Geophys. Res. Lett. 35, L01202 (2008)

G.B. Hansen, T.B. McCord, AGU Fall Meeting 2009, abstract \#P51E-1163 (2009)

P. Hartogh, E. Lellouch, R. Moreno, D. Bockelee-Morvan, N. Biver, T. Cassidy, M. Rengel, C. Jarchow, T. Cavalie, J. Crovisier, F.P. Helmich, M. Kidger, Astron. Astrophys. 532, L2+ (2011)

M.M. Hedman et al., Icarus 223(1), 105-130 (2013)

R. Helled, T. Guillot, Astrophys. J. 767, 113 (2013). doi:10.1088/0004-637X/767/2/113

R. Helled, J.D. Anderson, M. Podolak, G. Schubert, Astrophys. J. 726, 15 (2011). doi:10.1088/0004-637X/ $726 / 1 / 15$

A.R. Hendrix et al., Icarus 135(1), 79-94 (1998)

A.R. Hendrix et al., J. Geophys. Res. 104(E6), 14169-14178 (1999)

A.R. Hendrix et al., Europa's disk-resolved ultraviolet spectra: relationships with plasma flux and surface terrains. Icarus 212(2), 736-743 (2011)

B.E. Hesman, G.R. Davis, H.E. Matthews, G.S. Orton, Icarus 186, 342-353 (2007)

C.A. Hibbitts et al., Composition and distribution of nonice and trace materials on Ganymede as derived from Galileo observations, abstract n. 632. EPSC (2009)

C.A. Hibbitts et al., Distributions of $\mathrm{CO}_{2}$ and $\mathrm{SO}_{2}$ on the surface of Callisto. J. Geophys. Res. 105(E9), 22541-22558 (2000)

C.A. Hibbitts et al., $\mathrm{CO}_{2}$-rich impact craters on Callisto. J. Geophys. Res. 107(E10), 5084-5100 (2002)

C.A. Hibbitts et al., Carbon dioxide on Ganymede. J. Geophys. Res. 108(E5), 5036 (2003)

M.D. Hofstadter, B.J. Butler, Icarus 165, 168-180 (2003)

M.D. Hofstadter, D.O. Muhleman, Icarus 81, 396-412 (1989)

C.W. Hord et al., Galileo Ultraviolet Spectrometer experiment. Space Sci. Rev. 60(1-4), 503-530 (1992)

S.M. Hörst, V. Vuitton, R.V. Yelle, J. Geophys. Res. 113(E10), E10006 (2008)

W.-B. Hubbard, B. Militzer, Astrophys. J. 820, 80 (2016). doi:10.3847/0004-637X/820/1/80

H. Hussmann, F. Sohl, T. Spohn, Icarus 185, 258-273 (2006)

H. Hussmann, G. Choblet, V. Lainey et al., Space Sci. Rev. 153, 317-334 (2010)

H. Hussmann, C. Sotin, J. Lunine, in Treatise of Geophysics 2nd edn. (Elsevier, Amsterdam, 2015), pp. 605635. doi:10.1016/B978-0-444-53802-4.00178-0

L. Iess, R.A. Jacobson, M. Ducci, D.J. Stevenson, J.I. Lunine, J.W. Armstrong, S.W. Asmar, P. Racioppa, N.J. Rappaport, P. Tortora, Science 337(6093), 457-459 (2012). doi:10.1126/science.1219631

A. Ingersoll, A.R. Vasavada (Galileo Imaging Team), Nature 403, 630-632 (2000)

A.P. Ingersoll, T.E. Dowling, P.J. Gierasch, G.S. Orton, P.L. Read, A. Sanchez-Lavega, A.P. Showman, A.A. Simon-Miller, A.R. Vasavada, in Jupiter. The Planet, Satellites and Magnetosphere (2004), pp. 105-128

P. Irwin, N.A. Teanby, G.R. Davis, Icarus 203, 287-302 (2009)

P.G.J. Irwin, E. Lellouch, C. de Bergh, R. Courtin, B. Bezard, L.N. Fletcher, G.S. Orton, N.A. Teanby, S.B. Calcutt, D. Tice, J. Hurley, G.R. Davis, Icarus 227, 37-48 (2014)

M.A. Janssen, A.P. Ingersoll, M.D. Allison, S. Gulkis, A.L. Laraia, K.H. Baines, S.G. Edgington, Y.Z. Anderson, K. Kelleher, F.A. Oyafuso, Icarus 226, 522-535 (2013)

T.V. Johnson, J.I. Lunine, Saturn's moon Phoebe as a captured body from the outer solar system. Nature 435, 69-71 (2005)

R.E. Johnson et al., Astron. Astrophys. 123(2), 343-346 (1983)

J.S. Kargel, S.K. Croft, J.I. Lunine, J.S. Lewis, Icarus 89, 93-112 (1991)

E. Karkoschka, M.G. Tomasko, Icarus 211, 780-797 (2011)

S. Kattenhorn, L.M. Prockter, Nat. Geosci. 7, 762-767 (2014). doi:10.1038/ngeo2245

K.K. Khurana et al., Icarus 191(1), 193-202 (2007)

T.V.V. King, R.N. Clark, W.M. Calvin, D.M. Sherman, R.H. Brown, Science 255, 1551-1553 (1992)

R.L. Kirk, D.J. Stevenson, Icarus 69, 91-134 (1987)

M.G. Kivelson, Adv. Space Res. 33(11), 2061-2077 (2004)

M.G. Kivelson, K.K. Khurana, M. Volwerk, Icarus 157(2), 507-522 (2002)

M.G. Kivelson, K.K. Khurana, M. Volwerk, in Europa, ed. by R.T. Pappalardo, W.B. McKinnon, K.K. Khurana (University of Arizona Press, Tucson, 2009), pp. 545-570

O.L. Kuskov, V.A. Kronrod, Sol. Syst. Res. 39, 283 (2005). doi:10.1007/s11208-005-0043-0

M. Lambrechts, A. Johansen, Astron. Astrophys. 544, A32 (2012). doi:10.1051/0004-6361/201219127

J. Leconte, G. Chabrier, Astron. Astrophys. Rev. 540, A20 (2012). doi:10.1051/0004-6361/201117595

J. Leconte, F. Selsis, F. Hersant, T. Guillot, Astron. Astrophys. (2017). doi:10.1051/0004-6361/201629140

J. Leliwa-Kopystynski, M. Satorre, C. Santonja, Acta Geophys. 61, 1304-1321 (2013) 
E. Lellouch, B. Bezard, T. Fouchet, H. Feuchtgruber, T. Encrenaz, T. de Graauw, Astron. Astrophys. 370, 610-622 (2001)

E. Lellouch, B. Bezard, J.I. Moses, G.R. Davis, P. Drossart, H. Feuchtgruber, E.A. Bergin, R. Moreno, T. Encrenaz, Icarus 159, 112-131 (2002)

E. Lellouch, P. Hartogh, H. Feuchtgruber, B. Vandenbussche, T. de Graauw, R. Moreno, C. Jarchow, T. Cavalié, G. Orton, M. Banaszkiewicz, M.I. Blecka, D. Bockelée-Morvan, J. Crovisier, T. Encrenaz, T. Fulton, M. Küppers, L.M. Lara, D.C. Lis, A.S. Medvedev, M. Rengel, H. Sagawa, B. Swinyard, S. Szutowicz, F. Bensch, E. Bergin, F. Billebaud, N. Biver, G.A. Blake, J.A.D.L. Blommaert, J. Cernicharo, R. Courtin, G.R. Davis, L. Decin, P. Encrenaz, A. Gonzalez, E. Jehin, M. Kidger, D. Naylor, G. Portyankina, R. Schieder, S. Sidher, N. Thomas, M. de Val-Borro, E. Verdugo, C. Waelkens, H. Walker, H. Aarts, C. Comito, J.H. Kawamura, A. Maestrini, T. Peacocke, R. Teipen, T. Tils, K. Wildeman, Astron. Astrophys. 518, L152 (2010)

H.F. Levison, K.A. Kretke, M.J. Duncan, Nature 524, 322-324 (2015). doi:10.1038/nature14675

J.S. Lewis, Icarus 15, 174-185 (1971)

J. Licandro, N. Pinilla-Alonso, Astrophys. J. Lett. 630, L93 (2005)

N. Ligier, F. Poulet, J. Carter, R. Brunetto, F. Gourgeot, Astron. J. 151, 163 (2016). doi:10.3847/0004-6256/ $151 / 6 / 163$

B. Little, C.D. Anger, A.P. Ingersoll, A.R. Vasavada, D.A. Senske, H.H. Breneman, W.J. Borucki (The Galileo SSI Team), Icarus 142, 306-323 (1999)

K. Lodders, Astrophys. J. 591, 1220-1247 (2003). doi:10.1086/375492

K. Lodders, B. Fegley Jr., Icarus 112, 368-375 (1994)

R.M. Lopes, J.R. Spencer, Io After Galileo (Springer, Berlin, 2007). ISBN 978-3-540-34681-4

J.I. Lunine, Annu. Rev. Astron. Astrophys. 31, 217-263 (1993)

K.E. Mandt, O. Mousis, J. Lunine, D. Gautier, Astrophys. J. 788(2), L24 (2014)

M.S. Marley, P. Gomez, M. Podolak, J. Geophys. Res. 100, 23349-23354 (1995). doi:10.1029/95JE02362

A. Marten, D. Gautier, T. Owen, D.B. Sanders, H.E. Matthews, S.K. Atreya, R.P.J. Tilanus, J.R. Deane, Astrophys. J. 406, 285-297 (1993)

R.M. Mastrapa, S.A. Sandford, T.L. Roush, D.P. Cruikshank, C.M. Dalle Ore, Astrophys. J. 701(2), 13471356 (2009)

A. Matter, T. Guillot, A. Morbidelli, Planet. Space Sci. 57, 816-821 (2009). doi:10.1016/j.pss.2009.01.010

T.B. McCord, C. Sotin, J. Geophys. Res. $110(E 5)$, E05009 (2005)

T.B. McCord et al., Organics and other molecules in the surfaces of Callisto and Ganymede. Science 278, 271-275 (1997)

T.B. McCord et al., Non-water ice constituents in the surface material of the icy Galilean satellites from the Galileo near-infrared mapping spectrometer investigation. J. Geophys. Res. 103(E4), 8603-8626 (1998a)

McCord et al., Salts on Europa's surface detected by Galileo's Near Infrared Mapping Spectrometer. Science 280(5367), 1242 (1998b)

T.B. McCord et al., Hydrated salt minerals on Europa's surface from the Galileo Near-Infrared Mapping Spectrometer (NIMS) investigation. J. Geophys. Res. 104(E5), 11827-11852 (1999)

T.B. McCord et al., Thermal and radiation stability of the hydrated salt minerals epsomite, mirabilite, and natron under Europa enviromental conditions. J. Geophys. Res. 106(E2), 3311-3319 (2001)

T.B. McCord et al., Brines exposed to Europa surface conditions. J. Geophys. Res. 107(E1), 5004 (2002)

T.B. McCord, P. Hayne, J.-P. Combe (The Cassini VIMS Team), Icarus 194, 212-242 (2008). doi:10.1016/ j.icarus.2007.08.039

McCord et al., Hydrated minerals on Europa's surface: an improved look from the Galileo NIMS investigation. Icarus 209(2), 639-650 (2010)

M.A. McGrath, E. Lellouch, D.F. Strobel, P.D. Feldman, R.E. Johnson, in Jupiter, ed. by F. Bagenal, T. Dowling, W. McKinnon (Cambridge University Press, Cambridge, 2004)

W.B. McKinnon, in Proceedings of International Conference on Asteroids, Comets, Meteors, ACM 2002, 29 July-2 August 2002, Berlin, Germany, ed. by B. Warmbein. ESA, vol. SP-500 (ESA Publications Division, Noordwijk, 2002), pp. 29-38. ISBN 92-9092-810-7

W.B. McKinnon, F. Nimmo, T. Wong, P.M. Schenk, O.L. White, J.H. Roberts, J.M. Moore, J.R. Spencer, A.D. Howard, O.M. Umurhan, S.A. Stern, H.A. Weaver, C.B. Olkin, L.A. Young, K.E. Smith, R. Beyer, M. Buie, B. Buratti, A. Cheng, D. Cruikshank, C. Dalle Ore, R. Gladstone, W. Grundy, T. Lauer, I. Linscott, J. Parker, S. Porter, H. Reitsema, D. Reuter, S. Robbins, M. Showalter, K. Singer, D. Strobel, M. Summers, L. Tyler, M. Banks, O. Barnouin, V. Bray, B. Carcich, A. Chaikin, C. Chavez, C. Conrad, D. Hamilton, C. Howett, J. Hofgartner, J. Kammer, C. Lisse, A. Marcotte, A. Parker, K. Retherford, M. Saina, K. Runyon, E. Schindhelm, J. Stansberry, A. Steffl, T. Stryk, H. Throop, C. Tsang, A. Verbiscer, H. Winters, A. Zangari, New horizons geology, geophysics, and imaging theme team. Nature 534, 82-85 (2016) 
H.Y. McSween, J.P. Emery, A.S. Rivkin, M.J. Toplis, J. Castillo-Rogez, T.H. Prettyman, M.C. De Sanctis, C.M. Pieters, C.A. Raymond, C.T. Russell, Carbonaceous chondrites as analogs for the composition and alteration of Ceres. Meteorit. Planet. Sci. (2017, in press). doi:10.1111/maps.12947

F. Merlin, M.A. Barucci, E. Dotto, C. de Bergh, G. Lo Curto, Astron. Astrophys. 444, 977-982 (2005)

F. Merlin, A. Alvarez-Candal, A. Delsanti, S. Fornasier, M.A. Barucci, F.E. DeMeo, C. de Bergh, A. Doressoundiram, E. Quirico, B. Schmitt, Astron. J. 137, 315 (2009)

Y. Miguel, T. Guillot, L. Fayon, Astron. Astroph. 596, A114 (2016). doi:10.1051/0004-6361/201629732

B. Militzer, W.B. Hubbard, Astrophys. J. 774, 148 (2013). doi:10.1088/0004-637X/774/2/148

G. Mitri, A. Coustenis, G. Fanchini, A.G. Hayes, L. Iess, K. Khurana, J.-P. Lebreton, R.M. Lopes, R.D. Lorenz, R. Meriggiola, M.L. Moriconi, R. Orosei, C. Sotin, E. Stofan, G. Tobie, T. Tokano, F. Tosi, Planet. Space Sci. 104, 78-92 (2014)

H. Mizuno, Prog. Theor. Phys. 64, 544-557 (1980). doi:10.1143/PTP.64.544

N. Monga, S. Desch, Astrophys. J. 798, 9 (2015). doi:10.1088/0004-637X/798/1/9

M.H. Moore et al., Studies of proton-irradiated cometary-type ice mixtures. Icarus 54, 388-405 (1983)

J.M. Moore, C.R. Chapman, E.B. Bierhaus, R. Greeley, F.C. Chuang, J. Klemaszewski, R.N. Clark, J.B. Dalton, C.A. Hibbitts, P.M. Schenk, J.R. Spencer, R. Wagner, in Jupiter-The Planet, Satellites, and Magnetosphere, ed. by F. Bagenal, T. Dowling, W.B. McKinnon (Cambridge University Press, Cambridge, 2004), pp. 397-426. ISBN 0-521-81808-7

J.M. Moore, W.B. McKinnon, J.R. Spencer, A.D. Howard, P.M. Schenk, R.A. Beyer, F. Nimmo, K.N. Singer, O.M. Umurhan, O.L. White, S.A. Stern, K. Ennico, C.B. Olkin, H.A. Weaver, L.A. Young, R.P. Binzel, M.W. Buie, B.J. Buratti, A.F. Cheng, D.P. Cruikshank, W.M. Grundy, I.R. Linscott, H.J. Reitsema, C. Reuter Dennis, R. Showalter Mark, V.J. Bray, C.L. Chavez, C.J.A. Howett, T.R. Lauer, C.M. Lisse, A.H. Parker, S.B. Porter, S.J. Robbins, K. Runyon, T. Stryk, H.B. Throop, C.C. Tsang Constantine, J. Verbiscer Anne, A.M. Zangari, A.L. Chaikin, D.E. Wilhelms, F. Bagenal, G.R. Gladstone, T. Andert, J. Andrews, M. Banks, B. Bauer, J. Bauman, O.S. Barnouin, P. Bedini, K. Beisser, S. Bhaskaran, E. Birath, M. Bird, D.J. Bogan, A. Bowman, M. Brozovic, C. Bryan, M.R. Buckley, S.S. Bushman, A. Calloway, B. Carcich, S. Conard, C.A. Conrad, J.C. Cook, O.S. Custodio, C.M. Dalle Ore, C. Deboy, Z.J.B. Dischner, P. Dumont, A.M. Earle, H.A. Elliott, J. Ercol, C.M. Ernst, T. Finley, S.H. Flanigan, G. Fountain, M.J. Freeze, T. Greathouse, J.L. Green, Y. Guo, M. Hahn, D.P. Hamilton, S.A. Hamilton, J. Hanley, A. Harch, H.M. Hart, C.B. Hersman, A. Hill, M.E. Hill, D.P. Hinson, M.E. Holdridge, M. Horanyi, C. Jackman, R.A. Jacobson, D.E. Jennings, J.A. Kammer, H.K. Kang, D.E. Kaufmann, P. Kollmann, S.M. Krimigis, D. Kusnierkiewicz, J.E. Lee, K.L. Lindstrom, A.W. Lunsford, V.A. Mallder, N. Martin, D.J. McComas, R.L. McNutt, D. Mehoke, T. Mehoke, E.D. Melin, M. Mutchler, D. Nelson, J.I. Nunez, A. Ocampo, W.M. Owen, M. Paetzold, B. Page, J.W. Parker, F. Pelletier, J. Peterson, N. Pinkine, M. Piquette, S. Protopapa, J. Redfern, J.H. Roberts, G. Rogers, D. Rose, K.D. Retherford, M.G. Ryschkewitsch, E. Schindhelm, B. Sepan, M. Soluri, D. Stanbridge, A.J. Steffl, D.F. Strobel, M.E. Summers, J.R. Szalay, M. Tapley, A. Taylor, H. Taylor, G.L. Tyler, M.H. Versteeg, M. Vincent, R. Webbert, S. Weidner, G.E. Weigle, K. Whittenburg, B.G. Williams, K. Williams, S. Williams, W.W. Woods, E. Zirnstein, Science 351, 1284-1293 (2016)

J.I. Moses, E. Lellouch, B. Bezard, G.R. Gladstone, H. Feuchtgruber, M. Allen, Icarus 145, 166-202 (2000)

J.I. Moses, T. Fouchet, B. Bézard, G.R. Gladstone, E. Lellouch, H. Feucht- gruber, J. Geophys. Res., Planets 110, E08001 (2005)

O. Mousis, Y. Alibert, D. Hestroffer, U. Marboeuf, C. Dumas, B. Carry, J. Horner, F. Selsis, Mon. Not. R. Astron. Soc. 383, 1269-1280 (2008)

O. Mousis, L.N. Fletcher, J.-P. Lebreton et al., Planet. Space Sci. 104, 29-47 (2014)

O. Mousis, D.H. Atkinson, T. Spilker, E. Venkatapathy, J. Poncy, R. Frampton, A. Coustenis, K. Reh, J.-P. Lebreton, L.N. Fletcher, R. Hueso, M.J. Amato, A. Colaprete, F. Ferri, D. Stam, P. Wurz, S. Atreya, S. Aslam, D.J. Banfield, S. Calcutt, G. Fischer, A. Holland, C. Keller, E. Kessler, M. Leese, P. Levacher, A. Morse, O. Muñoz, J.-B. Renard, S. Sheridan, F.-X. Schmider, F. Snik, J.H. Waite, M. Bird, T. Cavalié, M. Deleuil, J. Fortney, D. Gautier, T. Guillot, J.I. Lunine, B. Marty, C. Nixon, G.S. Orton, A. SánchezLavega, Planet. Space Sci. 130, 80-103 (2016)

S. Nayakshin, R. Helled, A.C. Boley, Mon. Not. R. Astron. Soc. 440, 3797-3808 (2014). doi:10.1093/mnras/ stu473

N. Nettelmann, A. Becker, B. Holst, R. Redmer, Astrophys. J. 750, 52 (2012). doi:10.1088/0004-637X/750/ $1 / 52$

N. Nettelmann, R. Helled, J.J. Fortney, R. Redmer, Planet. Space Sci. 77, 143-151 (2013). doi:10.1016/j.pss. 2012.06.019

S. Newman et al., Photometric and spectral analysis of the distribution of crystalline and amorphous ices on Enceladus as seen by Cassini. Icarus 193, 397-406 (2008)

P.D. Nicholson et al., A close look at Saturn's rings with Cassini VIMS. Icarus 193(1), 182-212 (2008) 
H.B. Niemann, S.K. Atreya, G.R. Carignan, T.M. Donahue, J.A. Haberman, D.N. Harpold, R.E. Hartle, D.M. Hunten, W.T. Kasprzak, P.R. Mahaffy, T.C. Owen, S.H. Way, J. Geophys. Res. 103(12), 22831-22846 (1998)

H.B. Niemann, S.K. Atreya, J.E. Demick, D. Gautier, J.A. Haberman, D.N. Har- pold, W.T. Kasprzak, J.I. Lunine, T.C. Owen, F. Raulin, J. Geophys. Res., Planets 115, 12006 (2010)

F. Nimmo, B. Giese, R.T. Pappalardo, Geophys. Res. Lett. 30(5), 1233 (2003). doi:10.1029/2002GL016660

F. Nimmo, D.P. Hamilton, W.B. McKinnon, P.M. Schenk, R.P. Binzel et al., Nature 540, 94-96 (2016). doi:10.1038/nature20148

C.A. Nixon, P.G.J. Irwin, S.B. Calcutt, F.W. Taylor, R.W. Carlson, Icarus 150, 48-68 (2001)

K.S. Noll et al., Detection of ozone on Ganymede. Science 273(5273), 341-343 (1996)

G.S. Orton, J.H. Lacy, J.M. Achtermann, P. Parmar, W.E. Blass, Icarus 100, 541-555 (1992)

G.S. Orton, L.N. Fletcher, J.I. Moses, A.K. Mainzer, D. Hines, H.B. Hammel, F.J. Martin-Torres, M. Burgdorf, C. Merlet, M.R. Line, Icarus 243, 494-513 (2014a)

G.S. Orton, J.I. Moses, L.N. Fletcher, A.K. Mainzer, D. Hines, H.B. Hammel, J. Martin-Torres, M. Burgdorf, C. Merlet, M.R. Line, Icarus 243, 471-493 (2014b)

T. Owen, T. Encrenaz, Space Sci. Rev. 106, 121-138 (2003)

T. Owen, P.R. Mahaffy, H.B. Niemann, S. Atreya, M. Wong, Astrophys. J. Lett. 553, L77-L79 (2001)

R. Park, A.S. Konopliv, B.G. Bills, N. Rambaux, J.C. Castillo-Rogez, C.A. Raymond, A.T. Vaughan, A.I. Ermakov, M.T. Zuber, R.R. Fu, M.J. Toplis, C.T. Russell, A. Nathues, Nature 537, 515-517 (2016). doi:10.1038/nature 18955

M. Podolak, J.I. Podolak, M.S. Marley, Planet. Space Sci. 48, 143-151 (2000). doi:10.1016/S00320633(99)00088-4

J.B. Pollack, O. Hubickyj, P. Bodenheimer, J.J. Lissauer, M. Podolak, Y. Greenzweig, Icarus 124, $62-85$ (1996). doi:10.1006/icar.1996.0190

F. Postberg et al., Sodium salts in E-ring ice grains from an ocean below the surface of Enceladus. Nature 459(7250), 1098-1101 (2009)

F. Poulet et al., Compositions of Saturn's rings A, B, and C from high resolution near-infrared spectroscopic observations. Astron. Astrophys. 412, 305-316 (2003)

T.H. Prettyman, N. Yamashita, M.J. Toplis, H.Y. McSween, N. Schorghofer, S. Marchi, W.C. Feldman, J. Castillo-Rogez, O. Forni, D.J. Lawrence, E. Ammannito, B.L. Ehlmann, H.G. Sizemore, S.P. Joy, C.A. Polanskey, M.D. Rayman, C.A. Raymond, C.T. Russell, Science 355(6320), 55-59 (2017). doi:10.1126/science.aah6765

L.M. Prockter, R.M.C. Lopes, B. Giese, R. Jaumann, R.D. Lorenz, R.T. Pappalardo, G.W. Patterson, P.C. Thomas, E.P. Turtle, R.J. Wagner, Space Sci. Rev. 153, 63-111 (2010). doi:10.1007/s11214-010-9649-8

E. Quirico et al., Composition, physical state, and distribution of ices at the surface of Triton. Icarus 139(2), 159-178 (1999)

F. Raulin, Space Sci. Rev. 116, 471-487 (2005). doi:10.1007/s11214-005-1967-X

R.T. Reynolds, P.M. Cassen, Geophys. Res. Lett. 6(2), 121-124 (1979)

A.S. Rivkin, E.L. Volquardsen, B.E. Clark, Icarus 185, 563-567 (2006)

M. Roos-Serote, A.R. Vasavada, L. Kamp, P. Drossart, P. Irwin, C. Nixon, R.W. Carlson, Nature 405, 158-160 (2000)

E. Rosenblum, P. Garaud, A. Traxler, S. Stellmach, Astrophys. J. 731, 66 (2011). doi:10.1088/0004-637X/ $731 / 1 / 66$

L. Roth et al., Transient water vapor at Europa's south pole. Science 343(6167), 171-174 (2014)

T.L. Roush et al., Ice and minerals on Callisto-a reassessment of the reflectance spectra. Icarus 86, 355-382 (1990)

P. Rousselot, O. Pirali, E. Jehin, M. Vervloet, D. Hutsemekers, J. Manfroid, D. Cordier, M.-A. Martin-Drumel, S. Gruet, C. Arpigny, A. Decock, O. Mousis, Astrophys. J. 780(2), L17 (2014)

O. Ruesch, T. Platz, P. Schenk, L.A. McFadden, J.C. Castillo-Rogez et al., Science 353(6303), aaf4286 (2016). doi:10.1126/science.aaf4286

A. Sanchez-Lavega, T. del Rio-Gaztelurrutia, R. Hueso, J.M. Gomez-Forrellad, J.F. Sanz-Requena, J. Legarreta, E. Garcia-Melendo, F. Colas, J. Lecacheux, L.N. Fletcher, D. Barrado y Navascués, D. Parker, T. Akutsu, T. Barry, J. Beltran, S. Buda, B. Combs, F. Carvalho, P. Casquinha, M. Delcroix, S. Ghomizadeh, C. Go, J. Hotershall, T. Ikemura, G. Jolly, A. Kazemoto, T. Kumamori, M. Lecompte, P. Maxson, F.J. Melillo, D.P. Milika, E. Morales, D. Peach, J. Phillips, J.J. Poupeau, J. Sussenbach, G. Walker, S. Walker, T. Tranter, A. Wesley, T. Wilson, K. Yunoki (International Outer Planet Watch Team), Nature 475, 71-74 (2011)

J. Saur, S. Duling, L. Roth et al., J. Geophys. Res. Space Phys. 120(3), 1715-1737 (2015)

E.L. Schaller, M.E. Brown, Astrophys. J. Lett. 659, L61 (2007)

E.L. Schaller, M.E. Brown, Astrophys. J. Lett. 684, L107 (2008)

P. Schenk, F. Nimmo, Nat. Geosci. 9, 411-412 (2016) 
B.E. Schmidt, D.D. Blankenship, G.W. Patterson, P.M. Schenk, Nature (2011). doi:10.1038/nature10608

B.E. Schmidt et al., Nat. Geosci. 10, 338-343 (2017)

B. Schmitt, E. Quirico, F. Trotta, W.M. Grundy, in Solar System Ices, ed. by B. Schmitt, C. De Bergh, M. Festou (Kluwer Academic, Boston, 1998), pp. 199-240

N. Schorghofer, E. Mazarico, T. Platz, F. Preusker, S.E. Schröder, C.A. Raymond, C.T. Russell, Geophys. Res. Lett. 43, 6783-6789 (2016)

G. Schubert, F. Sohl, H. Hussmann, in Europa, ed. by R.T. Pappalardo, W.B. McKinnon, K.K. Khurana (University of Arizona Press, Tucson, 2009), pp. 353-368

F. Scipioni, F. Tosi, K. Stephan, G. Filacchione, M. Ciarniello, F. Capaccioni, P. Cerroni (the VIMS Team), Spectroscopic classification of icy satellites of Saturn I: identification of terrain units on Dione. Icarus 226, 1331-1349 (2013)

F. Scipioni, F. Tosi, K. Stephan, G. Filacchione, M. Ciarniello, F. Capaccioni, P. Cerroni (the VIMS Team), Spectroscopic classification of icy satellites of Saturn II: identification of terrain units on Rhea. Icarus 234, 1-16 (2014)

F. Scipioni, P. Schenk, F. Tosi, E. D’Aversa, R. Clark, J.-P. Combe, C.M. Dalle Ore, Deciphering sub-micron ice particles on Enceladus surface. Icarus 290, 183-200 (2017)

Y. Shinnaka, H. Kawakita, H. Kobayashi, M. Nagashima, D.C. Boice, Astrophys. J. 782, L16 (2014)

J.H. Shirley et al., Icarus 210(1), 358-384 (2010)

A. Showman, D.J. Stevenson, R. Mahlotra, Icarus 129, 367-383 (1997)

S. Simon et al., Magnetic signatures of a tenuous atmosphere at Dione. Geophys. Res. Lett. 38(15), L15102 (2011)

A.A. Simon-Miller, B. Conrath, P.J. Gierasch, R.F. Beebe, Icarus 145, 454-461 (2000)

K.N. Singer, S.A. Stern, Astrophys. J. Lett. 808, L50 (2016)

W.D. Smythe, T.B. McCord, A. Coradini, C.A. Hibbitts, F. Capaccioni, G.B. Hansen, G. Filacchione, R.N. Clark, P. Cerroni, R.H. Brown, K.H. Baines, G. Bellucci, J.-P. Bibring, B.J. Buratti, E. Bussoletti, M. Combes, D.P. Cruikshank, P. Drossart, V. Formisano, R. Jaumann, Y. Langevin, D.L. Matson, R.M. Nelson, P.D. Nicholson, B. Sicardy, C. Sotin, 29th Annual Lunar and Planetary Science Conference (1998), p. 1532

L.A. Soderblom, S.W. Kieffer, T.L. Becker, R.H. Brown, A.F. Cook, C.J. Hansen, T.V. Johnson, R.L. Kirk, E.M. Shoemaker, Science 250, 410-415 (1990)

W.B. Sparks, K.P. Hand, M.A. McGrath, E. Bergeron, M. Cracraft, S.E. Deustua, Astrophys. J. 829(2), 121 (2016)

J.R. Spencer, W.M. Calvin, M.J. Person, J. Geophys. Res. 100(E9), 19049-19056 (1995)

J.R. Spencer, A.C. Barr, L.W. Esposito, P. Helfenstein, A.P. Ingersoll, R. Jaumann, C.P. McKay, F. Nimmo, J.H. Waite, in Saturn from Cassini-Huygens, ed. by M.K. Dougherty, L.W. Esposito, S.M. Krimigis (Springer, Dordrecht, 2009), pp. 683-724. doi:10.1007/978-1-4020-9217-6_21

T. Spohn, G. Schubert, Icarus 161, 456-467 (2003)

R. Srama, Space Sci. Rev. 114(1-4), 465-518 (2004)

L.A. Sromovsky, P.M. Fry, J.H. Kim, Icarus 215, 292-312 (2011)

L.A. Sromovsky, K.H. Baines, P.M. Fry, Icarus 226, 402-418 (2013)

K. Stephan, Ph.D. Thesis, Freie Universität Berlin (2006). http://diss.fu-berlin.de/2007/806

K. Stephan, H. Hoffmann, K. Hibbitts, R. Wagner, R. Jaumann, EPSC 2009, abstract n. 633 (2009)

K. Stephan, R. Jaumann, R. Wagner, R.N. Clark, D.P. Cruikshank, C.A. Hibbitts, T. Roatsch, H. Hoffmann, R.H. Brown, G. Filiacchione, B.J. Buratti, G.B. Hansen, T.B. McCord, P.D. Nicholson, K.H. Baines, Icarus 206(2), 631-652 (2010)

K. Stephan, R. Jaumann, R. Wagner, R.N. Clark, D.P. Cruikshank, B. Giese, C.A. Hibbitts, T. Roatsch, K. Matz, R.H. Brown, G. Filacchione, F. Cappacioni, F. Scholten, B.J. Buratti, G.B. Hansen, P.D. Nicholson, K.H. Baines, R. Nelson, D.L. Matson, Planet. Space Sci. 61(1), 142-160 (2012)

S.T. Stewart, A. Seifter, A.W. Obst, Shocked $\mathrm{H}_{2} \mathrm{O}$ ice: thermal emission measurements and the criteria for phase changes during impact events. Geophys. Res. Lett. 35(23), L23203 (2008)

C.R. Stoker, Icarus 67, 106-125 (1986)

K. Sugiyama, K. Nakajima, M. Odaka, K. Kuramoto, Y.-Y. Hayashi, Icarus 229, 71-91 (2014)

D. Takir, J.P. Emery, H.Y. McSween, Icarus 257, 185-193 (2015)

B.D. Teolis, J.H. Waite, Icarus 272, 277-289 (2016)

B.D. Teolis, G.H. Joneas, P.F. Miles, R.L. Tokar, B.A. Magee, J.H. Waite et al., Science 330(6012), 18131815 (2010)

T. Terai, Y. Itoh, Y. Oasa, R. Furusho, J. Watanabe, Astrophys. J. 827, 65 (2016)

G. Tobie, O. Grasset, J.I. Lunine, A. Mocquet, C. Sotin, Icarus (2005). doi:10.1016/j.icarus.2004.12.007

G. Tobie, J.I. Lunine, C. Sotin, Nature 440(7080), 61-64 (2006)

R.L. Tokar, R.E. Johnson, M.F. Thomsen, E.C. Sittler, A.J. Coates, R.J. Wilson, D.T. Young, G.H. Jones, Geophys. Res. Lett. 39(3), L03105 (2012) 
F. Tosi, D. Turrini, A. Coradini, G. Filacchione (the VIMS Team), Mon. Not. R. Astron. Soc. 403(3), 11131130 (2010)

N. Turner, M. Choukroun, J. Castillo-Rogez, G. Bryden, Astrophys. J. Lett. 748, 92 (2012)

S. Vance, J.M. Brown, Geochim. Cosmochim. Acta 110, 176-189 (2013)

S. Vance, M. Bouffard, M. Choukroun, C. Sotin, Planet. Space Sci. 96, 62-70 (2014). doi:10.1016/j.pss. 2014.03.011

A. Vazan, R. Helled, M. Podolak, A. Kovetz, Astrophys. J. 829, 118 (2016). doi:10.3847/0004-637X/829/ 2/118

C. Visscher, J.I. Moses, S.A. Saslow, Icarus 209, 602-615 (2010)

J.H. Waite, W.S. Lewis, W.T. Kasprzak, V.G. Anicich, B.P. Block, T.E. Cravens, G.G. Fletcher, W.-H. Ip, J.G. Luhmann, R.L. Mcnutt, H.B. Niemann, J.K. Parejko, J.E. Richards, R.L. Thorpe, E.M. Walter, R.V. Yelle, Space Sci. Rev. 114(1-4), 113-231 (2004)

J.H. Waite, M.R. Combi, W.H. Ip, T.E. Cravens, R.L. McNutt, W. Kasprzak, R. Yelle, J. Luhmann, H. Niemann, D. Gell, B. Magee, G. Fletcher, J. Lunine, W.L. Tseng, Science 311(5766), 1419-1422 (2006)

J.H. Waite, D.T. Young, T.E. Cravens, A.J. Coates, F.J. Crary, B. Magee, J. Westlake, Science 316(5826), 870 (2007)

J.H. Waite, W.S. Lewis, B.A. Magee, J.I. Lunine, W.B. McKinnon, C.R. Glein, O. Mousis, D.T. Young, T. Brockwell, J. Westlake, M.-J. Nguyen, B.D. Teolis, H.B. Niemann, R.L. McNutt Jr., M. Perry, W.-H. Ip, Nature 460(7254), 487-490 (2009)

K.J. Walsh, A. Morbidelli, S.N. Raymond, D.P. O’Brien, A.M. Mandell, Nature 475, 206-209 (2011)

H.A. Weaver, M.W. Buie, B.J. Buratti, W.M. Grundy, T.R. Lauer, C.B. Olkin, A.H. Parker, S.B. Porter, M.R. Showalter, J.R. Spencer, S.A. Stern, A.J. Verbiscer, W.B. McKinnon, J.M. Moore, S.J. Robbins, P. Schenk, K.N. Singer, O.S. Barnouin, A.F. Cheng, C.M. Ernst, C.M. Lisse, D.E. Jennings, A.W. Lunsford, D.C. Reuter, D.P. Hamilton, D.E. Kaufmann, K. Ennico, L.A. Young, R.A. Beyer, R.P. Binzel, V.J. Bray, A.L. Chaikin, J.C. Cook, D.P. Cruikshank, C.M. Dalle Ore, A.M. Earle, G.R. Gladstone, C.J.A. Howett, I.R. Linscott, F. Nimmo, J.W. Parker, S. Philippe, S. Protopapa, H.J. Reitsema, B. Schmitt, T. Stryk, M.E. Summers, C.C.C. Tsang, H.H.B. Throop, O.L. White, A.M. Zangari, Science 351, aae0030 (2016)

S.J. Weidenschilling, J.S. Lewis, Icarus 20, 465-476 (1973)

R.A. West, K.H. Baines, A.J. Friedson et al., in Jovian Clouds and Haze, ed. by F. Bagenal, T.E. Dowling, W.B. McKinnon (2004), pp. 79-104

K. Wierzchos, M. Womack, G. Sarid, Astron. J. 153, 230 (2017)

M.H. Wong, G.L. Bjoraker, M.D. Smith, F.M. Flasar, C.A. Nixon, Planet. Space Sci. 52, 385-395 (2004a)

M. Wong, P. Mahaffy, S. Atreya, H. Niemann, T. Owen, Icarus 171(1), 153-170 (2004b)

G. Wuchterl, T. Guillot, J.J. Lissauer, in Giant Planet Formation, Protostars and Planets IV (2000), p. 1081 